\title{
An Explicit Description of the Global Attractor of the Damped and Driven Sine-Gordon Equation
}

\author{
Björn Birnir ${ }^{1,2 \star}$, Rainer Grauer ${ }^{1, \star \star}$ \\ 1 Department of Mathematics, University of California, Santa Barbara, CA 93106, USA \\ 2 The University of Iceland, Science Institute, 3 Dunhaga, Reykjavik 107, Iceland
}

Received: 17. July 1992/in revised form: 4 October 1993

\begin{abstract}
We prove that the size of the finite-dimensional attractor of the damped and driven sine-Gordon equation stays small as the damping and driving amplitude become small. A decomposition of finite-dimensional attractors in Banach space is found, into a part $\mathscr{B}$ that attracts all of phase space, except sets whose finitedimensional projections have Lebesgue measure zero, and a part $\mathscr{C}$ that only attracts sets whose finite-dimensional projections have Lebesgue measure zero. We describe the components of the $\mathscr{B}$-attractor and $\mathscr{C}$, which is called the "hyperbolic" structure, for the damped and driven sine-Gordon equation. $\mathscr{B}$ is low-dimensional but the dimension of $\mathscr{C}$, which is associated with transients, is much larger. We verify numerically that this is a complete description of the attractor for small enough damping and driving parameters and describe the bifurcations of the $\mathscr{B}$-attractor in this small parameter region.
\end{abstract}

\section{Contents}

1. Introduction . . . . . . . . . . . . . . . . . . . . . . . . . . . . . 539

2. Energy Estimates . . . . . . . . . . . . . . . . . . . . . . . . . . . . . 542

3. The Attractor of the Poincaré Map . . . . . . . . . . . . . . . . . . . . . . 551

4. Periodic Orbits . . . . . . . . . . . . . . . . . . . . . . . . . . . . . . 559

5. The B-Attractor and the Hyperbolic Structure . . . . . . . . . . . . . . . . . 572

Appendix. The Center Manifold of the Flappers . . . . . . . . . . . . . . . . . . 583

References . . . . . . . . . . . . . . . . . . . . . . . . 588

\section{Introduction}

Dissipative nonlinear wave equations on a finite domain possess finite-dimensional attractors and if the equations are driven by an autonomous force a complete

^ Partially supported by NSF grants DMS89-05770 and DMS89-03012

$\star \star$ Partially supported by an INCOR grant and a grant from the German Science Foundation 
qualitative analysis of these attractors is possible, see Babin and Vishik [BV], Temam $[\mathrm{T}]$ and Hale [Ha]. That is, the equations have Lyapunov functions and if the stationary solutions of the undamped and undriven equations are non-degenerate, then the attractors are regular and can be explicitly described, see Babin and Vishik [BV].

The damped sine-Gordon equation,

$$
u_{t t}-u_{x x}+\sin (u)=\varepsilon f(x, t)-\delta u_{t}, \quad 0 \leq x \leq L, \quad t \in \mathbb{R},
$$

driven by a time-periodic force on a finite spatial interval, and with periodic boundary conditions, models the phase-difference between two superconducting layers in a Josephson junction, see Pedersen [P]. It is a nonautonomous, dissipative, nonlinear wave equation, so the above methods do not directly apply. In addition, the unperturbed $(\varepsilon=0=\delta$ ) sine-Gordon equation, which is completely integrable, see Takhatajan and Faddeev [TF] and McKean [McK], has degenerate (non-hyperbolic) stationary solutions.

The attractor of the damped and driven nonlinear wave equations, with a discrete number of stationary solutions, is trivial, see Temam [T], with Dirichlet boundary conditions. Then it typically consists of a sink close to the origin $u=0$. In distinction, numerical experiments, see Lomdahl and Samuelsen [LS], Bishop et al. [BF1-4], Mazor et al. [MB], Reinisch et al. [RF], Taki et al. [Ta], Grauer and Birnir [GB], Overman et al. [O] and McLaughlin and Overman [MO], indicate that the damped and driven sine-Gordon equation has much more complicated solutions, that persists as time becomes large.

Ghidaglia and Temam [GT] proved that the sine-Gordon equation with Dirichlet boundary conditions has a finite-dimensional attractor. Temam [T] extended their results to include periodic boundary conditions. The estimates on the dimension of the attractor obtained by these authors indicated that the dimension could go to infinity as the damping went to zero. Presumably, this was caused by the complete integrability of the sine-Gordon equation itself. It has solutions which are infinite-dimensional tori. In this paper, we show that this not the case, that the size of the attractor in an appropriate complete metric space does not grow as $\varepsilon$ and $\delta$ become small, and for $\delta$ fixed as $\varepsilon \rightarrow 0$, the physically important part of the attractor is simply a periodic orbit close to the origin.

Many dissipative nonlinear PDE's share the feature of the damped and driven sineGordon equations that the estimates of the dimension of the attractor are much larger than the dimension observed in numerical simulations and physical experiments. This is particularly common among equations describing (weakly-) turbulent fluids, see for example Temam's [T] estimates for the dimension of the attractor of the twodimensional Navier-Stokes equation. The resolution of this apparent contradiction for the sine-Gordon equation is that the attractor $\mathscr{C}$ decomposes, $\mathscr{A}=\mathscr{B} \cup \mathscr{C}$, see Milnor [Mi] for the finite-dimensional case, into a (smaller) attractor $\mathscr{B}$ and a "hyperbolic" structure $\mathscr{C}$, so that $\mathscr{B}$ attracts all of phase space, except sets whose projection onto a finite-dimensional space, see Mañé $[\mathrm{M}]$, has Lebesgue measure zero. $\mathscr{C}$ only attracts sets whose finite-dimensional projection has Lebesgue measure zero and is associated with transient dynamics. Thus only $\mathscr{B}$ is relevant to the long-time asymptotics and since this decomposition of $\mathscr{f}$ is perfectly general this may resolve the problem for other nonlinear PDE's as well.

The bulk of the paper is devoted to proving the existence and finding the stability of the components of $\mathscr{B}$ and $\mathscr{C}$. The upshot is that with even spatial symmetry $\mathscr{B}$ has only three components: a "flat" periodic orbit close to the origin, and two breathers, one at the center, the other at the end of the spatial interval. $\mathscr{C}$ has many 
more components but they are all unstable and play a limited role in the dynamics. We resort to numerics to show that we get a complete description of $\mathscr{b}=\mathscr{B} \cup \mathscr{C}$, however, see Birnir [B4] for an analytic proof of this.

The parameter region that we consider is $0<\varepsilon, \delta<10^{-1}$, we will denote this by $\varepsilon, \delta=\mathscr{Q}\left(10^{-2}\right)$. In this region the breather components of $\mathscr{B}$ do not interact (interaction requires symmetries, a short spatial interval and strong driving, see Armbruster, Birnir and Buys $[\mathrm{ABB}]$ ) and their spatial structure remains the same. The temporal evolution, on the other hand, shows bifurcation cascades to strange attractors, that we describe. In Grauer and Birnir [GB], it was shown that the breathers have a six-dimensional center-manifold, where these bifurcations take place.

Bishop et al. [BF1, 2, 3, 4] and Overman et al. [O], [MO], have explored the sineGordon attractor numerically for the parameter region $10^{-1}<\varepsilon, \delta<1$. There the breathers interact and qualitatively the dynamics seem to be captured by a four mode truncation, see Bishop et al. [BF3, 4] and Kovacic and Wiggins [KW]. In principle, this ought to be provable by the center-manifold reduction of Grauer and Birnir [GB]. However, the resulting six-dimensional ODE's are unwieldy and the analysis remains open.

The sine-Gordon equation can be thought of as a model for the continuum limit of a chain of coupled pendula, Eq. 1.1 describes the damping and driving of this chain and $u(x, t)$ is the angle from the vertical down position of the pendulum at the point $x$ along the chain. This means that $u(x, t)$ must be construed modulo $2 \pi$ and in Sect. 2, we find a complete metric space, following Temam [T], where the mean of $u$ lies on a circle $S^{1}=[0,2 \pi)$. Factoring the decay rate $e^{-\delta t / 2}$, of the damped harmonic oscillator that we expect close to the origin, out of the energy of the sine-Gordon equation gives us a good estimate. In fact, we get an absorbing set in any Sobolev space with zero mean. We use this in Sect. 2 to get a compact and spatially smooth absorbing set.

We develop the theory of finite-dimensional attractors for the Poincare map of periodically driven dissipative nonlinear PDE's in Sect. 3, following Hale [Ha], Temam [T] and Babin and Vishik [BV]. We conclude that the Poincare map of the damped an driven sine-Gordon equation has a smooth finite-dimensional attractor whose size, in the complete metric space on which the energy induces a metric, depends only on $L$ the length of the pendulum chain and the ratio $\varepsilon / \delta$ of the driving and damping amplitudes.

We use Mañé's theorem, see Mañé [M], to state and prove the decomposition theorem for finite dimensional attractors in Sect. 3. This is a generalization of a theorem by Milnor [Mi] who proved the decomposition of attractors in finitedimensions.

The straight down position of the pendulum chain, $u \equiv 0$, perturbs into a stable periodic orbit called the flat. The straight up, $u \equiv \pi$, perturbs into an unstable periodic orbit. The existence of these periodic orbits is proven in Sect. 4 and their stability explored. In addition, we find finitely many periodic orbits which flap (along the $x$-direction) into both directions from the straight-up position. These are accordingly called flappers and their existence is proven in Sect. 4. The flappers are unstable but have in addition a one dimensional center-manifold, where bifurcations take place. The center-manifold analysis is placed in the Appendix but the results are stated in Sect. 4.

Section 5 contains the analysis of the breathers which are the most interesting parts of $\mathscr{B}$. They are born in pairs for $\varepsilon$ sufficiently large, one stable and belongs to $\mathscr{B}$, the other is unstable and belongs to $\mathscr{C}$. The existence of the breathers is proven and 
their stability found in Sect. 5. Then we verify numerically that the description of $\mathscr{B} \cup \mathscr{C}$ is complete and describe the bifurcations of $\mathscr{B}$. To summarize: The method gives a detailed description of $\mathscr{A}$ because it combines local and global analysis. For $\varepsilon$ sufficiently large and spatially even functions (we make this restriction on the initial data),

$\mathscr{B}=$ flat +2 breathers,

$\mathscr{C}=$ straight-up +2 breathers $+3 n$ flappers and their unstable manifolds,

$n=[L / 2 \pi]$ is the integer value and this describes the spatial structure. The components of $\mathscr{E}$ are connected through the unstable manifolds, which lie in $\mathscr{C}$ according to Proposition 4.6. Thus the spatial structure is regular. On the other hand the temporal evolution of the breathers can be very complicated, as discussed in Sect. 5. It is clear that $\mathscr{B}$ is low-dimensional, but the dimension of $\mathscr{C}$ is relatively large.

\section{Energy Estimates}

We need to find a priori estimates for the damped and driven sine-Gordon equation to prove global existence of solutions and show that all initial data is eventually absorbed by a set, that does not grow as the damping becomes small. Numerical experiments indicate that the pendulum chain can roll over continuously. However, since $u$ is an angle variable there is no difference between $u$ before and after the chain rolls over and we can compare the two values by subtracting the mean of $u$

$$
\widehat{u}=\frac{1}{L} \int_{0}^{L} u d x,
$$

$L$ being the length of the chain. Temam [T] introduced the spaces

$$
\bar{L}^{2}=\left\{u \in L^{2}, \widehat{u}=0\right\}, \quad \bar{H}^{1}=H^{1} \cap \bar{L}^{2} .
$$

We let $\bar{u}=u-\widehat{u}, \bar{v}=v-\widehat{v}$, denote the solution and its velocity with zero mean and work in the space

$$
\overline{\mathscr{H}}=\bar{H}^{1} \times \bar{L}^{2}[0, L] \times S^{1} \times \mathbb{R},
$$

to keep track of the mean and its velocity. This is not a linear space but it is a complete metric space with metric induced by

$$
\begin{aligned}
\mathscr{E}^{1 / 2} & =\left[\int_{0}^{L}\left\{\frac{\bar{v}^{2}+\widehat{v}^{2}+\bar{u}_{x}^{2}}{2}+1-\cos (u)\right\} d x\right]^{1 / 2} \\
& =\left[\int_{0}^{L}\left\{\frac{\bar{v}^{2}+\widehat{v}^{2}+\bar{u}_{x}^{2}}{2}+1-\cos (\bar{u}+\widehat{u})\right\} d x\right]^{1 / 2} .
\end{aligned}
$$

$\mathscr{E}^{1 / 2}$ is well defined on $\overline{\mathscr{H}}$ since it measures $\widehat{u} \bmod 2 \pi$, and it satisfies the triangle inequality by the addition formula for sine, since

$$
1-\cos (u)=2 \sin ^{2}\left(\frac{u}{2}\right) \text {. }
$$


We write the damped and driven sine-Gordon equation as a PDE

$$
\begin{aligned}
& \bar{u}_{t}=\bar{v} \\
& \bar{v}_{t}=\bar{u}_{x x}-\sin (\bar{u}+\widehat{u})+\widehat{\sin }(\bar{u}+\widehat{u})+\varepsilon \bar{f}(x, t)-\delta \bar{v},
\end{aligned}
$$

for the coordinates $(\bar{u}, \bar{v}) \in \bar{H}^{1} \times \bar{L}^{2}$, coupled to an ODE,

$$
\begin{aligned}
& \widehat{u}_{t}=\widehat{v} \\
& \widehat{v}_{t}=-\widehat{\sin }(\bar{u}+\widehat{u})+\varepsilon \widehat{f}(t)-\delta \widehat{v},
\end{aligned}
$$

for $(\widehat{u}, \widehat{v}) \in S^{1} \times \mathbb{R}$. This system is obtained by first taking the mean of the PDE to get the ODE and then subtracting the ODE from the PDE to get the PDE with mean zero. We use the notation $v=u_{t}$ and remark that the systems (2.1) and (2.2), and Eq. (1.1) are equivalent initial value problems for smooth data.

The PDE system is defined on $\bar{H}^{1} \times \bar{L}^{2}[0, L] \cap \mathscr{D}(W)$, where $\mathscr{D}(W)$ is the domain of the wave operator in $L^{2} \times L^{2}[0, L]$. We will show that these equations have an attractor in $\overline{\mathscr{H}}$ whereas the mean keeps increasing in $H^{1} \times L^{2}$. This was already shown in Temam [T] but here we use the "natural" metric induced by $\mathscr{E}^{1 / 2}$, on $\overline{\mathscr{H}}$, to get an absorbing set that does not increase in size as the damping $(\delta)$ decreases. The estimates are actually obtained for a slightly different "energy"

$$
E(u)=\int_{0}^{L}\left\{\frac{1}{2}\left[\left(\bar{v}+\frac{\delta}{2} \bar{u}\right)^{2}+u_{x}^{2}-\frac{\delta^{2} \bar{u}}{4}\right]+1-\cos (u)\right\} d x
$$

This quantity is well defined on $\overline{\mathscr{H}}$ for the same reason as $\mathscr{E}$ and its square root is also subadditive. It is not clear whether $E(u)$ is always positive definite but this is the case for $\delta$ not too large.

Lemma 2.1. The two energies $\mathscr{E}$ and $E$ induce equivalent metrics on $\overline{\mathscr{H}}$, since

$$
(1-\delta L / 2 \pi) \mathscr{E} \leq E \leq(1+\delta L / 2 \pi) \mathscr{E},
$$

for $\delta<2 \pi / L$.

Proof.

$$
\begin{aligned}
E & =\int_{0}^{L}\left\{\left[\left(\bar{v}+\frac{\delta \bar{u}}{2}\right)^{2}+\widehat{v}^{2}+u_{x}^{2}-\frac{\delta^{2}}{4} \bar{u}^{2}\right] / 2+1-\cos (u)\right\} d x \\
& =\int_{0}^{L}\left\{\left[\bar{v}^{2}+\delta \bar{v} \bar{u}+\widehat{v}^{2}+u_{x}^{2}\right] / 2+1-\cos (u)\right\} d x,
\end{aligned}
$$

where $\bar{u}=u-\widehat{u}$ is the solution with zero mean. Now by the Cauchy-Schwarz and Poincaré inequalities

$$
\begin{aligned}
\int_{0}^{L} \bar{v} \bar{u} d x & \leq|\bar{v}|_{2}|\bar{u}|_{2} \leq(L / \pi)|\bar{v}|_{2}\left|u_{x}\right|_{2} \\
& \leq(L / 2 \pi)\left(|\bar{v}|_{2}^{2}+\left|u_{x}\right|_{2}^{2}\right)
\end{aligned}
$$


since $a b \leq(1 / 2)\left(a^{2}+b^{2}\right)$. This shows, since $u_{x}=\bar{u}_{x}$, that

$$
\begin{aligned}
E & \leq(1+(\delta L) / 2 \pi) \int_{0}^{L}\left\{\left(\bar{v}^{2}+\widehat{v}^{2}+u_{x}^{2}\right) / 2+1-\cos (u)\right\} d x \\
& =(1+(\delta L) / 2 \pi) \mathscr{E} .
\end{aligned}
$$

The other inequality is similar.

Our main estimate uses the energy $E$ and combines the energies of the PDE (2.1) and the ODE (2.2) so as to avoid producing the factor $1 / \delta$, except in the combination $\varepsilon / \delta$. We use the notation

$$
|f|_{2}^{\infty}=\operatorname{ess} \sup _{t}|f|_{2}
$$

where $|f|_{2}$ denotes the $L^{2}$ norm. The idea is to factor out the (exponential) decay rate of harmonic oscillations, that we expect in a neighborhood of $(u, v)=(0,0)$, to get good estimates. This transformation

$$
\bar{u}=w e^{-\delta t / 2}, \quad \bar{v}=w_{t} e^{-\delta t / 2}-\frac{\delta \bar{u}}{2}
$$

allows us to factor the decay out of $E(u)$

$$
E^{1 / 2}(u)=E^{1 / 2}(w) e^{-\delta t / 2},
$$

and we are left with the energy

$$
E(w)=\int_{0}^{L}\left\{\frac{1}{2}\left(w_{t}^{2}+e^{\delta t} \widehat{u}_{t}^{2}+w_{x}^{2}-\frac{\delta^{2}}{4} w^{2}\right)+e^{\delta t}(1-\cos (u))\right\} d x
$$

Lemma 2.2. The energy $(E)$ of the damped and driven sine-Gordon equation satisfies the global estimate

$$
E^{1 / 2}(t) \leq E^{1 / 2}(0) e^{-\delta t / 2}+K_{0}\left(1-e^{-\delta t / 2}\right)
$$

where

$$
K_{0}=\sqrt{2}\left[L^{1 / 2}+L^{3 / 2} / 2 \pi+(\varepsilon / \delta)\left(|\bar{f}|_{2}^{\infty}+L^{1 / 2}|\widehat{f}|_{\infty}\right)\right]
$$

$L$ is the length of spatial interval, $\hat{f}$ is the mean-value of the driving force and $\bar{f}(x, t)=f(x, t)-\widehat{f}(t)$.

Remark 2.1. All the terms in the estimate have the dimension $L^{1 / 2}$ ( $L$ is length) except one which has the dimension $L^{3 / 2}$. However, this is dimensionally correct because if the (pendulum chain's) coupling constant, that was set to unity, is put back in, then the $L^{3 / 2}$ term gets the new dimension $L^{1 / 2}$. 
Proof. We first get an estimate for the mean value of the velocity $\widehat{u}_{t}$. Multiply the mean (2.2) of the damped and driven sine-Gordon equation

$$
\widehat{u}_{t t}+\delta \widehat{u}_{t}+\widehat{\sin (u)}=\varepsilon \widehat{f}
$$

by $\widehat{u}_{t}$, to get

$$
\begin{aligned}
\frac{\partial}{\partial t}\left(\frac{\widehat{u}_{t}^{2}}{2}\right)+\delta \widehat{u}_{t}^{2} & =[-\widehat{\sin (u)}+\varepsilon \widehat{f}] \widehat{u}_{t} \\
& \leq\left(1+\varepsilon|\widehat{f}|_{\infty}\right)\left|\widehat{u}_{t}\right| .
\end{aligned}
$$

The inequality is integrated to give

$$
\left|\widehat{u}_{t}(t)\right|_{2}^{2} \leq\left|\widehat{u}_{t}(0)\right|_{2}^{2} e^{-\delta t}+\frac{1}{\delta}\left(1+\varepsilon|\widehat{f}|_{\infty}\right)\left(1-e^{-\delta t}\right) .
$$

This is an unsatisfactory estimate because of the term $1 / \delta$. However, the equation above implies that

$$
\frac{\partial}{\partial t}\left[\left(e^{+\delta t / 2} \widehat{u}_{t}\right)^{2} / 2\right]=e^{\delta t}[-\widehat{\sin (u)}+\varepsilon \widehat{f}] \widehat{u}_{t},
$$

and this we will use below to get a good estimate. Consider the damped and driven sine-Gordon equation

$$
\bar{u}_{t t}-\bar{u}_{x x}+\delta \bar{u}_{t}+\sin (u)-\widehat{\sin (u)}=\varepsilon \bar{f}(x, t),
$$

where bar denotes zero mean. Now let

$$
\bar{u}=w e^{-\delta t / 2}, \quad \bar{u}_{t}=\left(w_{t}-\frac{\delta w}{2}\right) e^{-\delta t / 2},
$$

then the equation becomes

$$
w_{t t}-w_{x x}-\frac{\delta^{2}}{4} w+e^{\delta t / 2}(\sin (u)-\widehat{\sin (u)})=\varepsilon e^{\delta t / 2} \bar{f} .
$$

We multiply by $w_{t}$ and integrate $x$ over $[0, L]$ to get

$$
\begin{aligned}
& \frac{\partial}{\partial t} \int_{0}^{L}\left\{\left(w_{t}^{2}+w_{x}^{2}-\frac{\delta^{2}}{4} w^{2}\right) / 2\right\} d x+e^{\delta t / 2}\left[\int_{0}^{L} \sin (u) w_{t} d x-\widehat{\sin (u)} L \widehat{w}_{t}\right] \\
& \quad=\varepsilon e^{\delta t / 2} \int_{0}^{L} \bar{f} w_{t} d x .
\end{aligned}
$$

The change of coordinates gives

so that

$$
w_{t}=\left(\bar{u}_{t}+\frac{\delta}{2} \bar{u}\right) e^{\delta t / 2}
$$

$$
\begin{aligned}
\sin (u) e^{\delta t / 2} w_{t}= & e^{\delta t} \sin (u)\left(\bar{u}_{t}+\frac{\delta \bar{u}}{2}\right) \\
= & \frac{\partial}{\partial t}\left[e^{\delta t}(1-\cos (u))\right]-\delta e^{\delta t}(1-\cos (u))-e^{\delta t} \sin (u) \widehat{u}_{t} \\
& +\frac{\delta}{2} e^{\delta t} \sin (u) \bar{u}
\end{aligned}
$$


and

$$
\widehat{w}_{t}=e^{\delta t / 2} \int_{0}^{L}\left(\bar{u}_{t}+\frac{\delta}{2} \bar{u}\right) d x=0
$$

since $\bar{u}$ and $\bar{u}_{t}$ have mean zero. We substitute the above for $\sin (u) e^{\delta t / 2} w_{t}$ to get

$$
\begin{aligned}
& \frac{\partial}{\partial t} \int_{0}^{L}\left\{\left(w_{t}^{2}+w_{x}^{2}-\frac{\delta^{2}}{4} w^{2}\right) / 2+e^{\delta t}(1-\cos (u))\right\} d x \\
& =\delta \int_{0}^{L} e^{\delta t}(1-\cos (u)) d x-e^{\delta t / 2} \frac{\delta}{2} \int_{0}^{L} \sin (u) w d x \\
& \left.+L e^{\delta t} \widehat{\sin (u}\right)\left(\widehat{u}_{t}\right)+\varepsilon e^{\delta t / 2} \int_{0}^{L} \bar{f} w_{t} d x .
\end{aligned}
$$

Then we add the equality from above

$$
L \frac{\partial}{\partial t}\left[\left(e^{+\delta t / 2} \widehat{u}_{t}\right)^{2} / 2\right]=L e^{\delta t}[-\widehat{\sin (u)}+\varepsilon \widehat{f}] \widehat{u}_{t}
$$

to get the essential cancellation of the terms involving $\widehat{\sin (u)}$. The upshot is that

$$
\begin{aligned}
& \frac{\partial}{\partial t} \int_{0}^{L}\left\{\left(w_{t}^{2}+w_{x}^{2}-\frac{\delta^{2}}{4} w^{2}+e^{\delta t} \widehat{u}_{t}^{2}\right) / 2+e^{\delta t}(1-\cos (u))\right\} d x \\
& =\delta \int_{0}^{L} e^{\delta t}(1-\cos (u)) d x-e^{\delta t / 2} \frac{\delta}{2} \int_{0}^{L} \sin (u) w d x \\
& \quad+\varepsilon e^{\delta t / 2} \int_{0}^{L}\left(\bar{f} w_{t}+e^{\delta t / 2} \widehat{f}_{t}\right) d x,
\end{aligned}
$$

and we get the estimate

$$
\frac{\partial E(w)}{\partial t} \leq \sqrt{2} e^{\delta t / 2}\left[\left(L^{1 / 2}+L^{3 / 2} / 2 \pi\right) \delta+\varepsilon\left(|\bar{f}|_{2}^{\infty}+L^{1 / 2}|\widehat{f}|_{\infty}\right)\right] \times E^{1 / 2}(w),
$$

by the Cauchy-Schwarz and Poincaré inequalities, where

$$
E(w)=\int_{0}^{L}\left\{\left(w_{t}^{2}+w_{x}^{2}-\frac{\delta^{2}}{4} w^{2}+e^{\delta t} \widehat{u}_{t}^{2}\right) / 2+e^{\delta t}(1-\cos (u))\right\} d x
$$

The differential inequality is solved to give

$$
E^{1 / 2}(w) \leq E^{1 / 2}(w)(0)+K_{0}\left(e^{\delta t / 2}-1\right),
$$

where

$$
K_{0}=\sqrt{2}\left[\left(L^{1 / 2}+L^{3 / 2} / 2 \pi\right)+(\varepsilon / \delta)\left(|\bar{f}|_{2}^{\infty}+L^{1 / 2}|\widehat{f}|_{\infty}\right)\right]
$$


Finally the relationship

$$
E(w)(t)=e^{\delta t} E(u)
$$

gives 2.3 .

The local existence and uniqueness of solution to 2.1 and 2.2 is a straightforward application of a fixed point theorem since the nonlinearity is Lipschitz, see for example Reed [R], or Temam [T] and Lions [L] for a different method. Lemma 2.1 allows us to prove global existence and the existence of an absorbing set, see Coddington and Levinson [CL], in addition.

Proposition 2.1. The damped and driven sine-Gordon equation has a global solution, if $|\vec{f}|_{2}^{\infty}$ and $|\widehat{f}|_{\infty}<\infty$, and an absorbing set in

$$
\overline{\mathscr{H}}=\bar{H}^{1} \times \bar{L}^{2}[0, L] \times S^{1} \times \mathbb{R}
$$

defined by

$$
E^{1 / 2}(t) \leq \sqrt{2}\left[\left(L^{1 / 2}+L^{3 / 2} / 2 \pi\right)+(\varepsilon / \delta)\left(|\bar{f}|_{2}^{\infty}+L^{1 / 2}|\widehat{f}|_{\infty}\right)\right]+\kappa
$$

where $\kappa$ is an arbitrarily small constant.

Proof. The global existence follows from the a priori estimate 2.3. The energy is a continuous function of $t$, on $\bar{H}^{1} \times \bar{L}^{2}[0, L] \cap \mathscr{D}(W)$, and the a priori estimate allows us to extend the interval of a local existence to the whole $t$ axis, because the length of this interval only depends on the $\overline{\mathscr{H}}$ distance of the solution to the origin. Moreover,

$$
E^{1 / 2}(t) \leq K_{0}+\kappa
$$

when

$$
t \geq(2 / \delta) \ln \left[\left|E^{1 / 2}(0)-K_{0}\right| / \kappa\right],
$$

where $\kappa$ is arbitrarily small. This shows that 2.4 defines an absorbing set.

Remark 2.2. It is easy to see that the terms $\sqrt{2} L^{1 / 2}$ and $(\varepsilon / \delta)\left(|\vec{f}|_{2}^{\infty}+L^{1 / 2}|\widehat{f}|_{\infty}\right)$ must be present in $K_{0}$. The unperturbed sine-Gordon equation has two distinguished stationary solutions $(u, v)=(\pi, 0)$ and $(u, v)=(0,0)$ which perturb into nearby periodic orbits as will be proven below. $\mathscr{E}^{1 / 2}(\pi, 0)=\sqrt{2} L^{1 / 2}$ and the latter term above is the energy of a resonant periodic orbit around $(0,0)$, see Proposition 4.2 below. This leaves only the term $L^{3 / 2} / \sqrt{2} \pi$, which represents the pendulum chain's ability to store energy in spatially localized solutions, unaccounted for. This term is associated to the spatially localized breathers. It is not clear that $L^{3 / 2} / \sqrt{2} \pi$ is optimal for all values of $L$, but it can be verified numerically to be attained by the energy of perturbed breathers, whose existence is proven in Sect. 5, for a range of $L$ values.

We now generalize the estimates in Lemma 2.1 to every Sobolev space with zero mean $\bar{H}^{n}[0, L]=H^{n} \cap \bar{L}^{2} \cdot \bar{H}^{n}$ is closed linear subspace of $H^{n}$ and consequently a Hilbert space. First we show that the two energies that will provide the norm

$$
E_{n}=(1 / 2) \int_{0}^{L}\left\{\left[\partial_{x}^{n}\left(v+\frac{\delta}{2} u\right)\right]^{2}+\left(\partial_{x}^{n+1} u\right)^{2}-\frac{\delta^{2}}{4}\left(\partial_{x}^{n} u\right)^{2}\right\} d x, \quad n \geq 1
$$


and

$$
\mathscr{E}_{n}=(1 / 2) \int_{0}^{L}\left[\left(\partial_{x}^{n} v\right)^{2}+\left(\partial_{x}^{n+1} u\right)^{2}\right] d x, \quad n \geq 0,
$$

are equivalent, on $\bar{H}^{n}$.

Lemma 2.3. The energies $\mathscr{E}_{n}$ and $E_{n}, n \geq 1$, are equivalent on $\bar{H}^{n}$, for $\delta<2 \pi / L$,

$$
(1+\delta L / 2 \pi)^{-1} E_{n} \leq \mathscr{E}_{n} \leq(1-\delta L / 2 \pi)^{-1} E_{n} ;
$$

and $E_{n}, n \geq 1$, satisfies the global estimate

$$
\begin{aligned}
E_{n}^{1 / 2}(t) \leq & E_{n}^{1 / 2}(0) e^{-\delta t / 2}+\mathscr{P}_{1}\left\{L^{1 / 2}, E_{i}^{1 / 2}(0), K_{i}, i \leq n-1\right\} t e^{-\delta t / 2} \\
& +K_{n}\left(1-e^{-\delta t / 2}\right)
\end{aligned}
$$

where

$$
K_{n}=(1 / \delta) \mathscr{P}_{2}\left(L^{1 / 2}, K_{i}, i \leq n-1\right)+\sqrt{2}(\varepsilon / \delta)\left|\partial_{x}^{n} f\right|_{2}^{\infty},
$$

and $\mathscr{P}_{j}(j=1,2)$ are polynomials in the indicated constants.

Proof.

$$
\begin{aligned}
E_{n} & =(1 / 2) \int_{0}^{L}\left\{\left[\partial_{x}^{n}\left(v+\frac{\delta}{2} u\right)\right]^{2}+\left(\partial_{x}^{n+1} u\right)^{2}-\frac{\delta^{2}}{4}\left(\partial_{x}^{n} u\right)^{2}\right\} d x \\
& =(1 / 2) \int_{0}^{L}\left\{\left(\partial_{x}^{n} v\right)^{2}+\delta \partial_{x}^{n} v \partial_{x}^{n} u+\left(\partial_{x}^{n+1} u\right)^{2}\right\} d x \\
& \leq(1+\delta L / 2 \pi)(1 / 2) \int_{0}^{L}\left[\left(\partial_{x}^{n} u\right)^{2}+\left(\partial_{x}^{n+1} u\right)^{2}\right] d x \\
& =(1+\delta L / 2 \pi) \mathscr{E}_{n},
\end{aligned}
$$

by the same inequalities as in Lemma 2.1. The other inequality is similar.

We differentiate the damped and driven sine-Gordon equation $n$ times with respect to $x$,

$$
\partial_{t}^{2}\left(\partial_{x}^{n} u\right)-\partial_{x}^{n+2} u+\delta \partial_{t}\left(\partial_{x}^{n} u\right)=-\partial_{x}^{n-1}\left[(\cos (u)) u_{x}\right]+\varepsilon \partial_{x}^{n} f,
$$

make the change of variables $u=e^{-\delta t / 2} w$ and multiply the equation by $\partial_{t}\left(\partial_{x}^{n} w\right)$. Then an integration in $x$ gives

$$
\begin{aligned}
& \frac{\partial}{\partial t}(1 / 2) \int_{0}^{L}\left[\left(\partial_{t} \partial_{x}^{n} w\right)^{2}+\left(\partial_{x}^{n+1} w\right)^{2}-\frac{\delta^{2}}{4}\left(\partial_{x}^{n} w\right)^{2}\right] d x \\
& \quad=-\int_{0}^{L}\left(\partial_{x}^{n-1}\left(\cos (u) w_{x}\right)\right) \partial_{t}\left(\partial_{x}^{n} w\right) d x+\varepsilon e^{\delta t / 2} \int_{0}^{L} \partial_{x}^{n} f \partial_{t}\left(\partial_{x}^{n} w\right) d x .
\end{aligned}
$$

Now by the Poincaré inequality,

$$
\left|\partial_{x}^{n} u\right|_{\infty} \leq L^{1 / 2}\left|\partial_{x}^{n+1} u\right|_{2} \leq \sqrt{2} L^{1 / 2} E_{n}^{1 / 2}(t) .
$$


Moreover, if the energies $E_{k}, k \leq n-1$, satisfy the inequality

$$
\begin{aligned}
E_{k}^{1 / 2}(t) \leq & E_{k}^{1 / 2}(0) e^{-\delta t / 2} \\
& +\mathscr{P}_{1}^{k}\left(L^{1 / 2}, E_{i}^{1 / 2}(0), K_{i}, i \leq k-1\right) t e^{-\delta t / 2}+K_{k}\left(1-e^{-\delta t / 2}\right),
\end{aligned}
$$

where $\mathscr{P}_{1}^{k}$, is a polynomial in the indicated constants, then given $h>0$, there exists a time $t_{k}$ so that

$$
\left|\partial_{x}^{k} u\right|_{\infty} \leq \sqrt{2} L^{1 / 2} K_{k}+h
$$

for $t \geq t_{k}$. These uniform estimates and the Cauchy-Schwarz and Poincaré inequalities give the inequality

$$
2 \frac{\partial}{\partial t} E_{n}^{1 / 2}(w) \leq 2 \mathscr{P}_{1}^{n}+\mathscr{P}_{2}^{n} e^{\delta t / 2}+\sqrt{2} \varepsilon\left|\partial_{x}^{n} f\right|_{2}^{\infty} e^{\delta t / 2}
$$

Then integration and the relationship

$$
E_{n}(w)=E_{n}(u) e^{\delta t}
$$

give 2.5, with a straightforward induction argument.

Now we get global existence of solutions and the existence of an absorbing set in every Sobolev space, with zero mean.

Proposition 2.2. The damped and driven sine-Gordon equation has a global solution, if $\left|\partial_{x}^{n} \bar{f}\right|_{2}^{\infty}$ and $|\widehat{f}|_{\infty}<\infty$, and an absorbing set defined by

$$
\begin{aligned}
& E^{1 / 2}(t) \leq K_{0}+\kappa, \\
& E_{\jmath}^{1 / 2}(t) \leq K_{\jmath}+\kappa, \quad 1 \leq j \leq n,
\end{aligned}
$$

in every Sobolev space with zero mean

$$
\overline{\mathscr{H}}^{n}=\bar{H}^{n+1} \times \bar{H}^{n}[0, L] \quad n \geq 1,
$$

where $\kappa$ is an arbitrarily small number. If $n \geq 2$, this solution is strong and unique.

Proof. The global existence follows from the estimate 2.5 and the equivalence of the energies $E_{n}$ and $\mathscr{E}_{n}$ by the same argument as in Proposition 2.1. We define the norm on $\overline{\mathscr{H}}^{n}$ to be

$$
\|(\bar{u}, \bar{v})\|_{\overline{\mathscr{H}}^{n}} n=\sum_{k=0}^{n} \mathscr{E}_{k}^{1 / 2} .
$$

Notice that this is the usual Sobolev norm on the components $(\bar{u}, \bar{v})$. Then the equivalence of $E_{k}$ and $\mathscr{E}_{k}, 1 \leq k \leq n$, shows by 2.3 and 2.5 that there exists a $t_{0}$ such that

$$
\|(\bar{u}, \bar{v})(t)\|_{\mathscr{H}^{\prime}} \leq(1-\delta L / 2 \pi)^{-1 / 2} \sum_{\jmath=0}^{n} K_{\jmath}+\kappa
$$

for $t>t_{0}$, since $\mathscr{E}_{0} \leq \mathscr{E}$, and $\mathscr{E} \leq(1-\delta L / 2 \pi)^{-1 / 2} E(t)$. This defines an absorbing set in $\overline{\mathscr{H}}^{n}$. 
The reader may be wondering why we used $E(t)$ as a metric on $\overline{\mathscr{H}}$ but $\mathscr{E}_{k}(k=1, \ldots, n)$ (and $\mathscr{E}_{0}$ ) to give a norm on $\overline{\mathscr{H}}^{n}$. These are of course equivalent to $\mathscr{E}$ and $E_{k}(k=1, \ldots, n)$ respectively by Lemmas 2.1 and 2.3 , but we get a better estimate on $E(t)$ than $\mathscr{E}$ in $\overline{\mathscr{H}}$ and it is cleaner to use the $\mathscr{E}_{k}$ 's on $\overline{\mathscr{H}}^{n}$ because the mean appears in $E(t)$.

We cannot expect the solutions to a hyperbolic nonlinear PDE to have more smoothness than their initial data. But now we prove that there exists a global absorbing set that is spatially smooth. Moreover, we get a nice bound on its size in $\overline{\mathscr{H}}$.

Theorem 2.1. The damped and driven sine-Gordon equation has an absorbing set, if the driving function $f$ is spatially smooth, in

$$
C^{\infty} \times C^{\infty}[0, L] \times S^{1} \times \mathbb{R} .
$$

Its bound in $\overline{\mathscr{H}}$ is given by

$$
E^{1 / 2}(t) \leq \sqrt{2}\left[\left(L^{1 / 2}+L^{3 / 2} / 2 \pi\right)+(\varepsilon / \delta)\left(|\bar{f}|_{2}^{\infty}+L^{1 / 2}|\widehat{f}|_{\infty}\right)\right]+\kappa,
$$

where $\kappa$ is arbitrarily small.

Proof. Suppose the initial data of the damped and driven sine-Gordon equation 2.1 lies in $\overline{\mathscr{H}}^{n}$. We will split $\bar{u}$ into a part $z$ which remains in the $n^{\text {th }}$ Sobolev space with zero mean, but decays exponentially, and a part $w$ which lies in the $(n+1)^{\text {th }}$ Sobolev space with zero mean. Let

$$
\bar{u}=e^{-\delta t / 2}(w+z),
$$

where $w$ gets the nonlinearity and $z$ the initial data, see Hale [Ha] and Temam [T], so the $w$ equation has zero initial data

$$
\begin{aligned}
\partial_{t}^{2}\left(\partial_{x}^{n+1} w\right)-\partial_{x}^{n+3} w-\frac{\delta^{2}}{4} \partial_{x}^{n+1} w & =-\partial_{x}^{n}\left[\cos (u) \partial_{x}(w+z)\right]+\varepsilon e^{\delta t / 2} \partial_{x}^{n+1} f \\
\partial_{x}^{n} w(0) & =0, \quad \partial_{t} \partial_{x}^{n} w(0)=0
\end{aligned}
$$

and the $z$ equation is linear

$$
\begin{aligned}
& \partial_{t}^{2}\left(\partial_{x}^{n} z\right)-\partial_{x}^{n+2} z-\frac{\delta^{2}}{4} \partial_{x}^{n} z=0 \\
& \partial_{x}^{n} z(0)=\partial_{x}^{n} \bar{u}(0), \quad \partial_{t} \partial_{x}^{n} z(0)=\partial_{t} \partial_{x}^{n} \bar{u}(0) .
\end{aligned}
$$

The $(n+1)^{\text {th }}$ derivative on $w$ will be justified by its global estimate, but first we estimate $z$. We multiply the $z$ equation by $\partial_{t}\left(\partial_{x}^{n} z\right)$ and integrate with respect to $x$ to get the estimate

$$
\frac{\partial}{\partial t} \int_{0}^{L}\left\{\left[\left(\partial_{t} \partial_{x}^{n} z\right)^{2}+\left(\partial_{x}^{n+1} z\right)^{2}-\frac{\delta^{2}}{4}\left(\partial_{x}^{n} z\right)^{2}\right] / 2\right\} d x \leq 0 .
$$

An integration in $t$, then gives

$$
E_{n}\left(z e^{-\delta t / 2}\right) \leq E_{n}(0) e^{-\delta t / 2},
$$

or the $z$ part of $\bar{u}$ decays exponentially. The estimate of the $w$ part of $\bar{u}$ is very similar to the estimate in Lemma 2.3. We multiply by $\left(\partial_{t} \partial_{x}^{n+1} w\right)$, integrate in $x$, and use the 
Cauchy-Schwarz and Poincaré inequalities, along with the sup norm estimates 2.6, and the estimate of $z$ above, to show that

$$
E_{n+1}^{1 / 2}\left(e^{-\delta t / 2} w\right)(t) \leq \mathscr{P}_{1}^{n+1}\left(L^{1 / 2}, E_{i}^{1 / 2}(0), K_{i}, i \leq n\right) t e^{-\delta t / 2}+K_{n+1}\left(1-e^{-\delta t / 2}\right) .
$$

The important difference between this estimate and 2.5 is that the initial condition $E_{n+1}^{1 / 2}(0)$ is missing, because the initial data of $w$ is zero. This means that $w$ has global existence in $\overline{\mathscr{H}}^{n+1}$ if $E_{k}^{1 / 2}(z)(0), k \leq n$, is finite.

Now we bootstrap the above argument to show that

$$
\Pi_{w}[(\bar{u}, \bar{v})(t)] \in \overline{\mathscr{H}}^{m}, \quad \text { for any } m \in Z^{+},
$$

where $\Pi_{w}$ denotes the projection onto the (eventual) $w$ part. Then the Sobolev embedding theorem implies that

$$
\Pi_{w}(\bar{u}, \bar{v}) \in C^{m+1} \times C^{m}[0, L],
$$

for any integer $m$, or $\Pi_{w}(\bar{u}, \bar{v})(x, t)$ is smooth in $x$. The bound in $\overline{\mathscr{H}}$ follows from Proposition 2.1.

In Sect. 3 we will construct the attractor as the omega limit set, see Hartmann [H], of the absorbing set. That we have an absorbing set in $\overline{\mathscr{H}}^{1}$, which is compactly embedded in $\overline{\mathscr{H}}$, will be used to prove the compactness of the attractor. The regularity of attractors with autonomous driving and hyperbolic stationary solutions of the unperturbed equation is treated in Babin and Vishik [BV], see also Hale [Ha] and Temam [T]. We will see in Sect. 4 that not all the stationary solutions of sine-Gordon equation are hyperbolic. Solutions called flappers have a one-dimensional centermanifold. Moreover, with non-autonomous driving, which we are considering here, these methods fail and one must resort to Theorem 2.1 to prove regularity.

\section{The Attractor of the Poincaré Map}

A non-autonomous PDE acting on some complete metric space does not generate a semi-group. However, if the time dependence of the PDE is periodic, with period $T$, then the Poincaré map, see Hartmann [H] or Arnold [A],

$$
P(u, v)(t)=(u, v)(t+T)
$$

has the same properties as the time- $T$ map, see Hale [Ha], of an autonomous PDE. This means that the notions of limit sets, attractors, etc., carry over for the Poincare map. We collect the definitions of these structures for Poincare maps below.

Definition 3.1. A subset $X$ of a complete metric space is invariant under a map $P$ if it is both positively and negatively invariant,

$$
P^{n} X \subset X \text { and } X \subset P^{n} X,
$$

for $n \in \mathbb{Z}^{+}$.

Definition 3.2. If $X$ and $Y$ are two subsets of a complete metric space $\mathscr{H}$, with metric $d$ and

$$
d\left(P^{n} Y, X\right)=\sup \left\{d\left(P^{n} w, X\right): w \in Y\right\} \rightarrow 0,
$$

as $n \rightarrow \infty$, then $X$ attracts $Y$ (uniformly). 
Definition 3.3. $\mathscr{A}$ is an $A$-attractor if it attracts a neighborhood of itself (point-wise) and is invariant. If $\mathscr{A}$ is compact and attracts every bounded subset of $\mathscr{H}, \mathscr{A}$ is called a universal A-attractor.

Universal attractors are also called global attractors and the books of Hale [Ha], Temam [T] and Babin and Vishik [BV] can be consulted for more information about them.

Remark 3.1. Universal attractors are unique and maximal among attractors and invariant sets.

Now let

$$
M_{c}(X)=\inf \{\varepsilon \geq 0: X \text { can be covered by finitely many } \varepsilon \text {-balls }\} .
$$

$M_{c}$ is a measure of (non-)compactness, due to Kuratowski [K], and has the properties,

$$
\begin{aligned}
M_{c}(X \cup Y) & =\max \left[M_{c}(X), M_{c}(Y)\right], \\
M_{c}(X) & =M_{c}(\text { closure } X)
\end{aligned}
$$

and

$$
M_{c}(X)=0, \quad \text { if and only if } X \text { is precompact. }
$$

If $\mathscr{H}$ is a Banach space, then

$$
M_{c}(X+Y) \leq M_{c}(X)+M_{c}(Y) .
$$

All these properties of $M_{c}$, except the third one, are obvious. The third follows from the observation, that $X$ is totally bounded if $M_{c}(X)=0$, which means that $X$ is relatively compact.

Theorem 3.1. Assume that $X$ is an absorbing set of a complete metric space $\mathscr{H}$ under the map $P$ and that $\lim _{m \rightarrow \infty} M_{c}\left(\bigcup_{n \geq m} P^{n}(X)\right)=0$. Then the $\omega$-limit set of $X$

$$
\begin{aligned}
\omega(X) & =\bigcap_{n \geq 0} \overline{\bigcup_{m \geq n} P^{m}(X)} \\
& =\left\{w \in \mathscr{H}: \exists w_{n} \in X, n \rightarrow \infty \text { and } P^{n} w_{n} \rightarrow w\right\}
\end{aligned}
$$

is a universal attractor for $\mathscr{H}$. Proof. We show that $w(X)$ is nonempty, compact and invariant. If $K_{n}=\bigcup_{m \geq n} P^{m} X$
$K=\omega(X)$, then by the hypothesis

$$
M_{c}(K) \leq \lim _{n \rightarrow \infty} M_{c}\left(K_{n}\right)=0 .
$$

Recall that $M_{c}(X)=M_{c}$ (closure $X$ ). Thus $K$ is compact. If $w_{n} \in K_{n}$, then $\left\{w_{n}\right\}$ is precompact because

$$
M_{c}\left(\left\{w_{n}\right\}\right)=\max \left[M_{c}\left(\left\{w_{n}, n \leq N\right\}\right), M_{c}\left(\left\{w_{n}, n>N\right\}\right)\right] .
$$

$M_{c}\left(\left\{w_{n}, n>N\right\}\right) \leq M_{c}\left(K_{n}\right) \rightarrow 0$ when $n \rightarrow \infty . M_{c}\left(\left\{w_{n}, n \leq N\right\}\right)=0$, because a set with finitely many members is totally bounded. This says that $M_{c}\left(\left\{w_{n}\right\}\right)=0$. We pick a convergent subsequence, then $w=\lim _{n \rightarrow \infty} w_{n} \in K$, so $K$ is not empty. 
$K$ is obviously positively invariant. If $w \in K$, then there exists a sequence $z_{n}=P^{n} w_{n} \rightarrow w$ and $K_{m+n} \ni P^{m+n} w_{n}=P^{m} z_{n}$. This shows that

$$
P^{m} w=\lim _{n \rightarrow \infty} P^{m+n} w_{n} \in K .
$$

But $K$ is also negatively invariant. If $P^{n} w_{n} \rightarrow w$, then $\left\{P^{n-m} w_{n}\right\}$ has a convergent subsequence and if $z=\lim _{n \rightarrow \infty} P^{n-m} w_{n}$, then

$$
w=P^{m} z \in P^{m} K .
$$

Finally we show that $K$ attracts bounded subsets of $\mathscr{H}$. If not then there exist $w_{n} \in X$, such that

$$
d\left(P^{n} w_{n}, K\right) \geq \varepsilon>0 .
$$

But $\left\{P^{n} w_{n}\right\}$ has a convergent subsequence

$$
w=\lim _{n \rightarrow \infty} P^{n} w_{n} \in K
$$

which is a contradiction.

Remark 3.2. The $\omega$-limit set $\omega(X)$ under a continuous Poincaré map of a vector field is connected if $\mathscr{H}$ is a Banach space and $X$ is convex, see Babin and Vishik [BV].

We now state sufficient conditions on the map $P$ that imply $P$ has a universal attractor.

Proposition 3.1. Let $\mathscr{H}^{n}$ be compactly embedded in $\mathscr{H}^{n-1}$, where $\mathscr{H}^{k}, k=n-1, n$, are Banach spaces and assume that $P: \mathscr{H}^{k} \rightarrow \mathscr{H}^{k}$ can be split

$$
P=Z+W
$$

such that $Z$ and $W$ satisfy the following conditions:

i. $Z$ contracts bounded subsets of $\mathscr{H}^{k}, k=n-1, n$, uniformly, to zero.

ii. $W: \mathscr{H}^{n-1} \cap \mathscr{D}(P) \rightarrow \mathscr{H}^{n}$, is continuous, where $\mathscr{D}(P)$ is the domain of $P$, and if $X \subset \mathscr{H}^{n-1} \cap \mathscr{D}(P)$ is bounded, then $W(X)$ is bounded in $\mathscr{H}^{n}$.

Now if $X \subset \mathscr{H}^{n-1}$ is an absorbing set for $P$, then

$$
\lim _{m \rightarrow \infty} M_{c}\left(\bigcup_{n \geq m} P^{n}(X)\right)=0 \text {. }
$$

Proof. The splitting of $P$ defines a sequence of nonlinear maps $W_{m}: \mathscr{H}^{n-1} \cap$ $\mathscr{D}(P) \rightarrow \mathscr{H}^{n}$, which we will show have the same property (ii) as $W$. The $W_{m}$ 's can be defined by iteration

$$
\begin{aligned}
W_{m+1}(x) & =Z W_{m}(x)+W\left(Z^{m} x+W_{m}(x)\right), \quad m \geq 2, \\
W_{1}(x) & =W(x), \quad W_{0}(x)=0, \quad x \in X .
\end{aligned}
$$

This iteration formula shows that $W_{m+1}: \mathscr{H}^{n-1} \cap \mathscr{D}(P) \rightarrow \mathscr{H}^{n}$, if $W_{m}$ : $\mathscr{H}^{n-1} \cap \mathscr{D}(P) \rightarrow \mathscr{H}^{n}$, and that $W_{m+1}(X)$ is bounded in $\mathscr{H}^{n}$, if $W_{m}(X)$ is bounded in $\mathscr{H}^{n-1}$, because $Z$ is a contraction on $\mathscr{H}^{n-1}$. Notice that $W_{1}=W$.

The rest is straightforward.

$$
\bigcup_{n \geq m} P^{n}(X)=P^{m}\left(\bigcup_{n \geq 0} P^{n}(X)\right) \subseteq Z^{m}\left(\bigcup_{n \geq 0} P^{n}(X)\right)+W_{m}\left(\bigcup_{n \geq 0} P^{n}(X)\right)
$$


and

$$
M_{c}\left(\bigcup_{n \geq m} P^{n}(X)\right) \leq M_{c}\left(Z^{m}\left(\bigcup_{n \geq 0} P^{n}(X)\right)\right)+M_{c}\left(W_{m}\left(\bigcup_{n \geq 0} P^{n}(X)\right)\right) .
$$

But

$$
M_{c}\left(Z^{m}\left(\bigcup_{n \geq 0} P^{n}(X)\right)\right) \leq \theta^{m} M_{c}(X), \quad 0<\theta<1
$$

because $Z$ is a contraction and $\bigcup_{n \geq 0} P^{n}(X) \subset X$. Moreover,

$$
M_{c}\left(W_{m}\left(\bigcup_{n \geq 0} P^{n}(X)\right)\right)=0
$$

because $W_{m}\left(\bigcup_{n \geq 0} P^{n}(X)\right)$ is bounded in $\mathscr{H}^{n}$ and consequently compact in $\mathscr{H}^{n-1}$, by (ii). We conclude that

$$
\lim _{m \rightarrow \infty} M_{c}\left(\bigcup_{n \geq m} P^{n}(X)\right) \leq \lim _{m \rightarrow \infty} \theta^{m} M_{c}(X)=0 .
$$

Proposition 3.1 applies to the time- $T$ map of damped and driven nonlinear wave equations, see Babin and Vishik [BV] who treat the autonomous case, and the Poincaré maps of the non-autonomous equations. It implies that such equations have a universal attractor by Theorem 3.1. We apply Theorem 2.1 to prove that the periodically (in time) driven and damped sine-Gordon equation, with periodic (in space) boundary conditions, has a universal attractor. This was originally proven by Ghidaglia and Temam [GT] for Dirichlet boundary conditions and Temam [T] for periodic boundary conditions, the improvement here is that the size (in the $\overline{\mathscr{H}}$ metric) of the attractor does not grow with $1 / \delta$.

Proposition 3.2. The Poincaré map $P$ of the damped and driven sine-Gordon equation has a universal A-attractor $\omega(X)$ in the complete metric space,

$$
\overline{\mathscr{H}}=\bar{H}^{1} \times \bar{L}^{2}[0, L] \times S^{1} \times \mathbb{R},
$$

and in every Sobolev space with zero mean,

$$
\overline{\mathscr{H}}^{n}=\bar{H}^{n+1} \times \bar{H}^{n}[0, L], \quad n \in \mathbb{Z}^{+} .
$$

Consequently, $\omega(X)$ is spatially smooth.

Proof. First we consider the Hilbert spaces

$$
\overline{\mathscr{H}}^{n-1}=\bar{H}^{n} \times \bar{H}^{n-1}[0, L], \quad n \in \mathbb{Z}^{+} .
$$

The hypotheses in Proposition 3.1, describes the splitting $u=z+w$, in Theorem 2.1, where $z$ gives a linear contraction, but $w$ is a solution to a nonlinear PDE with initial value zero. The corresponding mappings $Z$ and $W$ satisfy conditions (i) and (ii) in Proposition 3.1. In addition, $\overline{\mathscr{H}}^{n}$ is compactly embedded in $\overline{\mathscr{H}}^{n-1}$. Consequently,

$$
\lim _{m \rightarrow \infty} M_{c}\left(\bigcup_{k \geq m} P^{k}\left(X_{n-1}\right)\right)=0
$$

in $\overline{\mathscr{H}}^{n-1}$ and Theorem 3.1 says that $\omega\left(X_{n-1}\right)$ is a universal attractor in $\overline{\mathscr{H}}^{n-1}$. 
We have an absorbing set $X_{0}$, when $n=0$, in the complete metric space

$$
\overline{\mathscr{H}}=\bar{H}^{1} \times \bar{L}^{2}[0, L] \times S^{1} \times \mathbb{R}
$$

defined by $E^{1 / 2}(\bar{u}, \bar{v}, \widehat{u}, \widehat{v}) \leq K_{0}+\kappa$. This gives a bound on the mean velocity $\widehat{v}$ and the bounded subset $\widehat{X}_{0}$ of $S^{1} \times \mathbb{R}^{1}$, defined by $L^{1 / 2}|\widehat{v}| / \sqrt{2} \leq K_{0} . \widehat{X}_{0}$ is obviously compact. Consequently,

$$
\lim _{m \rightarrow \infty} M_{c}\left(\bigcup_{k \geq m} P^{k}\left(\widehat{X}_{0}\right)\right)=0
$$

where the Poincare map is defined by the ODE 2.2. This implies that $\omega\left(\widehat{X}_{0}\right)$ is a universal attractor in $S^{1} \times \mathbb{R}$. The argument for $\overline{\mathscr{H}}=\bar{H}^{1} \times \bar{L}^{2}[0, L]$ is the same as above so the attractor of the Poincare map of the coupled PDE (2.1) and ODE (2.2) is

$$
\omega\left(X_{0}\right)=\omega_{\mathrm{PDE}}\left(\bar{X}_{0}\right) \times \omega_{\mathrm{ODE}}\left(\widehat{X}_{0}\right) .
$$

Finally, we define by Proposition 2.2 an absorbing set in

$$
\overline{\mathscr{H}}^{n}=\bar{H}^{n+1} \times \bar{H}^{n}[0, L]
$$

by the (energy) condition:

$$
(\bar{u}, \bar{v}) \in X_{n}, \text { if } E_{j}^{1 / 2}(t) \leq K_{j}+\kappa, \quad 0 \leq j \leq n,
$$

then $\omega\left(X_{n}\right)$ is a universal attractor in $\overline{\mathscr{H}}^{n}$. But the norm in $\overline{\mathscr{H}}^{k}$ is stronger than the norm in $\overline{\mathscr{H}}^{m}, k \geq m$, consequently $\omega\left(X_{k}\right)$ is also a universal attractor in $\overline{\mathscr{H}}^{m}$. However the universal attractor is unique, so $\omega\left(X_{k}\right)=\omega(X), \forall k$. The Sobolev embedding theorem now says that $\omega(X)$ is spatially smooth.

$A$-attractors are unsatisfactory objects both from mathematical and physical standpoints. They contain hyperbolic fixed points and periodic orbits which only attract their stable manifolds, which are of "measure" zero. In addition, the $A$-attractors contain the unstable manifolds of hyperbolic objects. These manifolds are frequently associated with transient dynamics and although these are sometimes of physical interest, most of the time they are not. The real interest is in the long-time dynamics; in numerical computations, the first few thousand iterates are typically ignored, and we would like to dissociate the "true" attractor from this extraneous "hyperbolic structure." This was done by Milnor [Mi] in finite dimensions and we present below an amplification of his ideas.

The idea is to define the real (basic) attractor to consist only of those parts of the $A$-attractor that attract sets of measure greater than zero.

Let $\mathscr{H}$ be a separable complete metric space possessing an $A$-attractor $\mathscr{A}$ of a continuous map $P: \mathscr{H} \rightarrow \mathscr{H}$. The (topological) dimension of $\mathscr{C}$ is bounded by the Hausdorff dimension which is in turn bounded by the limit capacity, or fractal dimension,

$$
\operatorname{dim} \mathscr{b} \leq \operatorname{dim}_{H} \mathscr{b} \leq c(\mathscr{A}) .
$$

A theorem by Mañé [M] tells us that if the limit capacity of $\mathscr{A}$, is finite there exists a finite dimensional subspace which gives an honest, but perhaps "flattened," picture of $\mathscr{l}$. 
Theorem 3.2. If $\mathscr{H}$ is a Banach space, $P: \mathscr{H} \rightarrow \mathscr{H}$ a map satisfying the hypothesis of Proposition 3.1, and $\mathscr{b}$ the universal A-attractor of $\mathscr{H}$ under $P$, then

$$
c(\mathscr{A})<\infty \text {. }
$$

Moreover, if $Y$ is any linear subspace of $\mathscr{H}$ with dimension

$$
\operatorname{dim}(Y) \geq 2 c(\mathscr{A})+1,
$$

then there exists a residual set $V$ of all projections of $\mathscr{H}$ onto $Y$, such that $\Pi \in V$ is one to one on $\mathcal{A}$.

The finiteness of $c(\mathscr{A})$ is proven in Temam [T] and Babin and Vishik [BV]. The latter statement in the theorem is proven in Mañé [M], see Sauer et al. [SYC] and references therein for more information.

We use Mañé's theorem to define the basic attractor $\mathscr{B}$ which captures the essential part of $\mathscr{C}$ and separates from it the extraneous structure $\mathscr{C}$. The realm of attraction $\mathscr{B}(\mathscr{A})$ of a set $\mathscr{A}$ consists of all $x$ such that $\omega(x) \in \mathscr{C}$, if it is open, it coincides with the basin of attraction of $\mathscr{b}$.

Definition 3.4. An attractor $\mathscr{B}(\subseteq \mathscr{C})$ is called a $B$-attractor if it satisfies the two conditions:

(1) The projection of its realm of attraction $\Pi(\mathscr{B}(\mathscr{B}))$ has positive Lebesgue measure in $Y$, for all $\Pi \in V$.

(2) There exists no strictly smaller attractor $\mathscr{B}^{\prime} \subset \mathscr{B}$ such that the realms agree $\mathscr{B}(\mathscr{B})=\mathscr{B}\left(\mathscr{B}^{\prime}\right)$, up to sets $U$ whose projection $\Pi(U)$ has zero Lebesgue measure in $Y$, for all $\Pi \in V$.

The first condition says that any point in the projection of $\mathscr{B}$ counts and the second condition says that $\mathscr{B}$ is minimal in this respect. Our probe space $Y$, see Sauer et al. [SYC], will be chosen to be $\mathbb{R}^{n}$. We can now state the decomposition theorem.

Theorem 3.3. Let $A$ be a universal attractor of a continuous map on a Banach space $\mathscr{H}$ and $V$ the residual set of projections $\Pi: \mathscr{H} \rightarrow \mathbb{R}^{n}$, where $n \geq 2 c(\mathscr{A})+1$, which are one to one on $\mathscr{b}$. Then $\mathscr{A}$ can be decomposed into a $B$-attractor $\mathscr{B}$ and a "hyperbolic" structure $\mathscr{C}$,

$$
\mathscr{C}=\mathscr{B} \cup \mathscr{C},
$$

with realms of attraction $\mathscr{B}(\mathscr{B})$ and $\mathscr{B}(\mathscr{C})$, such that for every $\Pi \in V$ and every open set $U \subset \mathscr{R}(\mathscr{B}), \Pi(U)$ has positive Lebesgue measure in $\mathbb{R}^{n}$; whereas for every sufficiently small $U \subset \mathscr{B}(\mathscr{C}) \backslash \mathscr{B}(\mathscr{B})$ there exists a $\Pi \in V$ such that the Lebesgue measure of $\Pi(U)$ in $\mathbb{R}^{n}$ is zero.

Proof. Let $\Pi \in V$ be a projection $\Pi: \mathscr{H} \rightarrow \mathbb{R}^{n}$ which is one to one on $\mathscr{A}$. Let $D$ be a closed bounded subset of $\mathbb{R}^{n}$ with Lebesgue measure $\mu(D)<0$, such that $\Pi(\mathscr{A}) \subset D$ and let $\left\{U_{k}, k \in \Omega\right\}$ be a countable open covering of $D$. We choose a subset $\Omega^{\prime}$ of the index set $\Omega$ such that

$$
U_{k} \cap \Pi(\omega(x))=\phi, \quad \text { for all } k \in \Omega^{\prime},
$$

except for $x \in U \subset \mathscr{H}$ such that $\Pi(U)$ is of Lebesgue measure zero in $\mathbb{R}^{n}$. That is, we do not exclude open sets $U_{k}$ containing $\omega$-limit sets of sets in $\mathscr{H}$ whose projection has Lebesgue measure zero. Now let

$$
U=\bigcup_{k \in \Omega^{\prime}} U_{k}
$$


then

$$
B=D \backslash U
$$

is a closed set which contains $\Pi(\omega(x))$ for almost every $\Pi(x) \in D$. Then we refine the covering and consider the set $B_{\Pi}$ which is the intersection over all such refinements of the covering. This set is non-empty because $\Pi(\mathscr{B}(\mathscr{b}))=\mathbb{R}^{n}$ so that $\mathscr{A}$ must have at least one connected component $\mathscr{b}_{1}$ such that interior of $\mathscr{B}\left(\mathscr{C}_{1}\right)=U_{1}$ is non-empty. Now by the open mapping theorem $\Pi\left(U_{1}\right)$ is open, thus it has Lebesgue measure $\mu\left(\Pi\left(U_{1}\right)\right)>0$. Moreover, $B_{\Pi}$ is independent of the covering of $D$, and since $\Pi$ is one to one on $\mathscr{A}$ and $B_{\Pi} \subset \Pi(\mathscr{C}), \Pi^{-1}$ is well defined on $B_{\Pi}$. We define the $B$-attractor to be

$$
\mathscr{B}=\bigcap_{\Pi \in V} \Pi^{-1}\left(B_{\Pi}\right)
$$

$\mathscr{B}$ is closed by construction and compact because $\mathscr{B} \subset \mathscr{A}$ and $\mathscr{A}$ is compact. It is non-empty by the open mapping theorem because if $z$ attracts an open subset of $\mathscr{B}\left(\mathscr{A}_{1}\right)$ then $\Pi(z) \in B_{\Pi}$ for every $\Pi \in V$. Finally

$$
\mu(\Pi(\mathscr{B}(\mathscr{B}))) \geq\left(\Pi\left(U_{1}\right)\right)>0, \quad \text { for all } \Pi \in V,
$$

and by the same argument as above $\mu(\Pi(U))>0$ for every open set $U \subset \mathscr{R}(\mathscr{B})$ and every $\Pi \in V$. Thus since $\mathscr{B}$ is also minimal with respect to Condition 2, in Definition 3.4 , by construction, $\mathscr{B}$ is a $B$-attractor and it must contain all $B$-attractors of the map.

The "hyperbolic" structure is defined to be

$$
\mathscr{C}=\bigcup_{\Pi \in V} \Pi^{-1}\left(C_{\Pi}\right)
$$

where $C_{\Pi}=\Pi(\mathscr{\ell}) \backslash B_{\Pi}$. By the construction of $B_{\Pi}$,

$$
\mu\left(\Pi\left[\mathscr{R}\left(\Pi^{-1}\left(C_{\Pi}\right) \backslash \Pi^{-1}\left(B_{\Pi}\right)\right)\right]\right)=0, \text { for all } \Pi \in V .
$$

We denote $\mathscr{C}_{\Pi}=\Pi^{-1}\left(C_{\Pi}\right), \mathscr{B}_{\Pi}=\Pi^{-1}\left(B_{\Pi}\right)$, and first prove that

$$
\begin{aligned}
\mathscr{B}(\mathscr{C}) \backslash \mathscr{B}(\mathscr{B}) & =\mathscr{R}\left(\bigcup_{\Pi \in V} \mathscr{C}_{\Pi}\right) \backslash \mathscr{B}\left(\bigcap_{\Pi \in V} \mathscr{B}_{\Pi}\right) \\
& \subseteq \bigcup_{\Pi \in V} \mathscr{B}\left(\mathscr{C}_{\Pi} \backslash \mathscr{B}_{\Pi}\right) .
\end{aligned}
$$

Let $x \in \mathscr{B}\left(\bigcup_{\Pi \in V} \mathscr{C}_{\Pi}\right) \backslash \mathscr{R}\left(\bigcap_{\Pi \in V} \mathscr{B}_{\Pi}\right)$ then there exists a sequence $x_{n} \in \mathscr{C}_{\Pi_{n}}$ such that $P^{n} x-x_{n} \rightarrow 0$ as $n \rightarrow \infty$, where $P^{n} x$ are iterates of $x$ under the map. The sequence $\left\{x_{n}\right\} \subset \bigcup_{\Pi \in V} \mathscr{C}_{\Pi} \subset \mathscr{C}$ has a convergent subsequence $x_{n_{k}} \rightarrow y \in \mathscr{C}$ as $k \rightarrow \infty$ by the compactness of $\mathscr{A}$. Now $y \notin \bigcap_{\Pi \in V} \mathscr{B}_{\Pi}$ because that would imply $x \in \mathscr{R}\left(\bigcap_{\Pi \in V} \mathscr{B}_{\Pi}\right)$. Thus $y \in \bigcup_{\Pi \in V} \mathscr{C}_{\Pi} \backslash \bigcap_{\Pi \in V} \mathscr{B}_{\Pi}=\bigcup_{\Pi \in V}\left(\mathscr{C}_{\Pi} \backslash \mathscr{B}_{\Pi}\right)$ by De Morgan's law, so $y \in\left(\mathscr{C}_{\Pi} \backslash \mathscr{B}_{\Pi}\right)$ for some $\Pi$, but this implies that $x \in \mathscr{B}\left(\mathscr{C}_{\Pi} \backslash \mathscr{B}_{\Pi}\right)$ for some $\Pi$, or $x \in \bigcup_{\Pi \in V} \mathscr{B}\left(\mathscr{C}_{\Pi} \backslash \mathscr{B}_{\Pi}\right)$. Now if

$$
U \subset \mathscr{B}(\mathscr{C}) \backslash \mathscr{B}(\mathscr{B}) \subseteq \bigcup_{\Pi \in V} \mathscr{B}\left(\mathscr{C}_{\Pi} \backslash \mathscr{B}_{\Pi}\right)
$$


is sufficiently small, then by the continuity of the map $U$ must be attracted to a connected component of $\mathscr{C}_{\Pi} \backslash \mathscr{B}_{\Pi}$ for some $\Pi$. Then

$$
\mu(\Pi(U)) \leq \mu\left(\Pi\left[\mathscr{B}\left(\mathscr{C}_{\Pi} \backslash \mathscr{B}_{\Pi}\right)\right]\right)=0 .
$$

Corollary 3.1. The A-attractor of the damped and driven sine-Gordon equation decomposes into a $B$-attractor $\mathscr{B}$ that attracts of all phase space, except sets $U$ with associated Lebesgue measure in $\mathbb{R}^{n} \mu(\Pi(U))=0$, for all $\Pi \in V$; and a "hyperbolic" structure $\mathscr{C}$ consisting of hyperbolic orbits and their unstable manifolds.

Proof. Let $X \subset \overline{\mathscr{H}}$ be the absorbing set from Theorem 2.1. Then $\mathscr{f}=\omega(X)=$ $\omega(\bar{X}) \times \omega(\widehat{X})$ is the global attractor of the Poincare map of the damped and driven sine-Gordon equation from Proposition 3.2, where $X=\bar{X} \times \widehat{X}, \bar{X} \subseteq \bar{H}^{1} \times \bar{L}^{2}[0, L]$ and $\widehat{X} \subset S^{1} \times \mathbb{R}$. By Theorem 3.3, $\overline{\mathscr{C}}=\omega(\bar{X})$ can be decomposed; $\overline{\mathscr{A}}=\overline{\mathscr{B}} \cup \overline{\mathscr{C}}$, because $\bar{H}^{1} \times \bar{L}^{2}$ is a Hilbert space, into a $B$-attractor and a "hyperbolic structure" respectively. Moreover, since $\widehat{\mathscr{b}}=\omega(\widehat{X})$ lies in a finite-dimensional space (the cylinder) it can be decomposed by Milnor's theorem [Mi]; $\widehat{\mathscr{A}}=\widehat{\mathscr{B}} \cup \widehat{\mathscr{C}}$, into a $B$-attractor and a "hyperbolic" structure respectively. Now let $\mathscr{B}=\overline{\mathscr{B}} \times \widehat{\mathscr{B}}$ and $\mathscr{C}=\overline{\mathscr{C}} \times \hat{\mathscr{C}}$, then,

$$
\mathscr{A}=\mathscr{B} \cup \mathscr{C}
$$

It remains to show that $\mathscr{B}$ is a $B$-attractor and $\mathscr{C}$ a "hyperbolic structure." First let $U \subset \mathscr{R}(\mathscr{B})$ be an open set, then $U=\bar{U} \times \widehat{U}$, where $\bar{U} \subset \mathscr{R}(\overline{\mathscr{B}})$ and $\widehat{U} \subset \mathscr{R}(\widehat{\mathscr{B}})$. The Lebesgue product measure is

$$
\mu(\Pi(\bar{U}) \times \widehat{U})=\bar{\mu}(\Pi(\bar{U})) \widehat{\mu}(\widehat{U})>0
$$

since $\overline{\mathscr{B}}$ and $\widehat{\mathscr{B}}$ are $B$-attractors and therefore,

$$
\bar{\mu}(\Pi(\bar{U}))>0 \text { and } \widehat{\mu}(\widehat{U})>0 .
$$

This shows that $\mathscr{B}$ is also a $B$-attractor. Secondly, if $U \subset \mathscr{R}(\mathscr{C}) \backslash \mathscr{R}(\mathscr{B})$ is sufficiently small and $U=\bar{U} \times \widehat{U}$, where $\bar{U} \subset \mathscr{R}(\overline{\mathscr{C}}) \backslash \mathscr{R}(\overline{\mathscr{B}})$ and $\widehat{U} \subset \mathscr{R}(\widehat{\mathscr{C}}) \backslash \mathscr{R}(\widehat{\mathscr{B}})$, then either there exists $\Pi \in V$ such that

$$
\bar{\mu}(\Pi(\bar{U}))=0 \quad \text { or } \quad \widehat{\mu}(\widehat{U})=0,
$$

where $\Pi(\bar{U}) \times \widehat{U} \subseteq \mathbb{R}^{n-2} \times S^{1} \times \mathbb{R}$. In either case, the product measure vanishes

$$
\mu(\Pi(\bar{U}) \times \widehat{U})=\bar{\mu}(\Pi(\bar{U})) \widehat{\mu}(\widehat{U})=0 .
$$

In the remainder of the paper we describe the components of $\mathscr{B}$ and $\mathscr{C}$, for the damped and driven sine-Gordon equation.

Definition 3.5. A compact subset $\mathscr{B}$ of $\mathscr{H}$ is a minimal $B$-attractor if it is a $B$ attractor and there exists no strictly smaller $B$-attractor $\mathscr{B}^{\prime} \subset \mathscr{B}$. 
It is not possible in general to decompose the $B$-attractor into minimal $B$-attractors, see Milnor [Mi], but if

$$
\mathscr{B}=\bigcup_{\imath=1}^{n} \mathscr{B}_{\imath}, \quad n<\infty,
$$

where the $\mathscr{B}_{\imath}$ 's are minimal, then the whole space decomposes

where

$$
\mathscr{H}=\bigcup_{i=1}^{n} \mathscr{R}\left(\mathscr{B}_{i}\right)
$$

$$
\mathscr{B}\left(\mathscr{B}_{i}\right) \cap \mathscr{B}\left(\mathscr{B}_{j}\right)=\phi \quad i \neq j,
$$

up to sets who have a projection of measure zero in $\mathbb{R}^{n}$.

Remark 3.3. The terminology should be taken with a grain of salt because $\mathscr{C}$ contains unstable manifolds which are not hyperbolic. Moreover, $A$-attractors and $B$-attractors is a cumbersome terminology and it would simplify things to call $\mathscr{A}$, that attracts everything, the global attractor and $\mathscr{B}$, that attracts "almost" everything, the basic attractor.

\section{Periodic Orbits}

The recipe for the construction of the $B$-attractor and the "hyperbolic structure" $\mathscr{C}$ for small perturbation parameters, is to start with the stationary solutions of the unperturbed PDE. These should turn into periodic orbits with time-periodic driving and the stable periodic orbits belong to the $B$-attractor, the unstable ones to $\mathscr{C}$ for generic values of the perturbation parameters. In this section we prove the existence of periodic orbits perturbing from the stationary solutions of the sine-Gordon equation and find their stability. In addition we must consider what happens to the simplest time-periodic orbits of the unperturbed equation and this will be done in Sect. 5 .

The stationary solutions of the unperturbed sine-Gordon equation perturb into three different kinds of periodic orbits under the influence of periodic driving and damping. The first one is the origin $(0,0)$ which perturbs to a stable periodic orbit, that we will call "the flat," for moderate driving amplitudes. The second one is the "straightup" position $(\pi, 0)$ which perturbs to an unstable periodic orbit. The third type of periodic orbits arise from elliptic stationary solutions of the unperturbed sine-Gordon equation which flap in the two direction from the straight-up position and are called "flappers." They are unstable but have a marginal direction. Recall that $\mathscr{D}(W)$ denotes the domain of the wave operator in $L^{2} \times L^{2}[0, L]$.

Proposition 4.1. The unperturbed sine-Gordon equation has three types of stationary solutions in $\mathscr{D}(W) \subset H^{1} \times L^{2}[0, L]$ :

(1) The flat $(u, v)=(0,0)$,

(2) The straight-up, $(u, v)=(\pi, 0)$, and

(3) $n=[L / 2 \pi]$, circles of flappers $\left(u_{m}\left(x+x_{0}\right), 0\right), x_{0} \in S^{1}$,

$$
u_{m}(x)=4 \tan ^{-1}\left[\sqrt{k} s n\left(\frac{2 m x}{k+1}, k\right)\right]+\pi,
$$

where $s n$ is the Jacobi elliptic function and $k=(L / 2 \pi n)-1,1 \leq m \leq n$. 
Proof. The stationary solutions are determined by the (spatial) inverted pendulum equation

$$
u_{x x}-\sin (u)=0
$$

with the energy

$$
u_{x}^{2} / 2+1+\cos (u)=h .
$$

The flat $\left(u, u_{x}\right)=(0,0)$ and the straight up $\left(u, u_{x}\right)=(\pi, 0)$ are obvious solutions of these equations, with energy $h=2$ and $h=0$, respectively. In addition, when $h<2$, we get (translation) circles of solutions

$$
u\left(x+x_{0}\right)=4 \tan ^{-1}\left[\sqrt{k} \operatorname{sn}\left(\frac{2\left(x+x_{0}\right)}{(k+1)}, k\right)\right]+\pi, \quad x_{0} \in S^{1},
$$

where $h=8 k /(1+k)^{2}, k>0$ and $s n$ is the Jacobi elliptic function, see du Val [PV], with modulus $k$. These solutions flap in opposite directions from $\pi$ and are called flappers. They are periodic in $x$, and $2 /(1+k)=4 \pi m / L$, because we can choose the real period of $s n$ to be $2 \pi$ and their number is the integer value $n=[L / 2 \pi]$, since 1 is the frequency of the linear oscillation around $(\pi, 0)$, so that $2 \pi n / L \leq 1$. Solutions with $h>2$ have a twist. They are either nonperiodic kinks or kink-antikinks which do not have a second derivative and fit, therefore, not into $\mathscr{D}(W)$.

We linearize the sine-Gordon equation around the stationary solutions to examine their stability. The linear system can be written

$$
w_{t}=A w,
$$

where

$$
A=\left(\begin{array}{cc}
0 & 1 \\
\partial^{2} / \partial x^{2}+q & -\delta
\end{array}\right)
$$

and

$$
q=\left\{\begin{array}{c}
-1 \\
1 \\
-2\left(\wp\left(x+i \omega_{2}+x_{0}\right)-e_{2}\right)+1
\end{array}\right.
$$

for the stationary solution

$$
(u, v)=\left\{\begin{array}{c}
(0,0) \\
(\pi, 0) \\
\text { a flapper }
\end{array}\right.
$$

$\wp$ is a Weierstrass $p$-function, see du Val [PV], $e_{2}$ one of its stationary value and $i \omega_{2}$ is half its imaginary period. The transformation in Lemma 2.2 that gave the good energy estimates enables us to decompose the linear system into a conservative and a dissipative part. Let

$$
\Delta=\left(\begin{array}{cc}
1 & 0 \\
-\delta / 2 & 1
\end{array}\right), \quad \Delta^{-1}=\left(\begin{array}{cc}
1 & 0 \\
\delta / 2 & 1
\end{array}\right)
$$

then

$$
A=\Delta(\bar{A}-(\delta / 2) I) \Delta^{-1}
$$

where

$$
\bar{A}=\left(\begin{array}{cc}
0 & 1 \\
\frac{\partial^{2}}{\partial x^{2}}+q+\delta^{2} / 4 & 0
\end{array}\right) .
$$



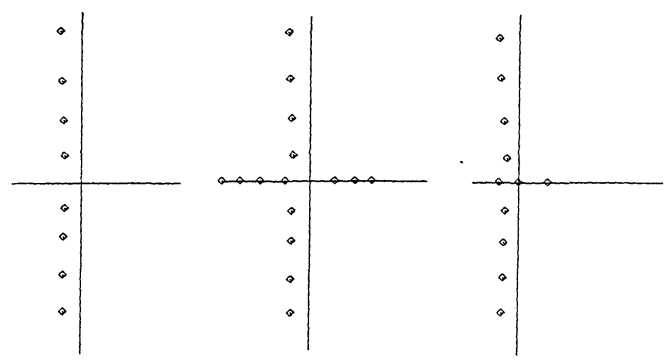

Fig. 4.1. The spectrum of the flat, the straight-up and the flappers

Lemma 4.1. The spectrum of the damped undriven sine-Gordon equation linearized about,

(1) The flat, $(u, v)=(0,0)$, is

$$
\lambda_{ \pm j}^{(1)}=-\delta / 2 \pm i\left[1-\delta^{2} / 4+4 \pi^{2} j^{2} / L^{2}\right]^{1 / 2}, \quad j \in \mathbb{Z}^{+} \cup\{0\} .
$$

(2) The straight up, $(u, v)=(\pi, 0)$, is

$$
\lambda_{ \pm j}^{(2)}=-\delta / 2 \pm\left[1+\delta^{2} / 4-4 \pi j^{2} / L^{2}\right]^{1 / 2} .
$$

(3) The flappers, $(u, v)=\left(u_{m}(x), 0\right), m \leq n=[L / 2 \pi]$, is

$$
\begin{aligned}
& \lambda_{ \pm 1}^{(3)}=-\delta / 2 \pm\left((m / n)^{2}\left(e_{1}-e_{3}\right)+\delta^{2} / 4\right)^{1 / 2}, \\
& \lambda_{-2}^{(3)}=-\delta, \quad \lambda_{2}^{(3)}=0, \\
& \lambda_{ \pm 3}^{(3)}=-\delta / 2 \pm i\left((m / n)^{2}\left(e_{3}-e_{2}\right)-\delta / 4\right)^{1 / 2}, \\
& \lambda_{ \pm j}^{(3)}=-\delta / 2 \pm i\left((m / n)^{2}\left(\mu_{j}-\left(1+2 e_{2}\right)\right)-\delta^{2} / 4\right)^{1 / 2}, \quad j \geq 4,
\end{aligned}
$$

where the $e_{i}$ 's are the stationary values of the Weierstrass p-function

$$
e_{1}-e_{3}=1-\frac{8 \pi n}{L}\left(1-\frac{2 \pi n}{L}\right), \quad e_{3}-e_{2}=\frac{8 \pi n}{L}\left(1-\frac{2 \pi n}{L}\right),
$$

and the $\mu_{j}$ 's are the simple periodic and anti-periodic eigenvalues of the Hill's operator with a Lamé potential

$$
-d^{2} / d x+2 \wp\left(m\left(x+i \omega_{2}\right)\right), \text { periods } L / 2, \quad 2 i \omega_{2} .
$$

Remark 4.1. The eigenvalues $\lambda_{j}^{(1)}$ in the first case above lie on a line parallel to the imaginary axis, but in the distance $\delta / 2$ to it, in the left-half plane. In the second case, $n+1$ of the eigenvalues $\lambda_{j}^{(2)}$ lie on the positive real axis, $n+1$ on the negative real axis and the rest on the line above in the left half-plane. In the third case $\lambda_{1}^{(3)}$ is on the positive real axis $\lambda_{-1}^{(3)}$ and $\lambda_{-2}^{(3)}$ on the negative real axis, $\lambda_{2}^{(3)}$ at zero and the rest on the line above in the left-half plane, see Fig. 4.1. 
Proof. The spectra in the flat and the straight-up cases are found by a straightforward computation using the identity 4.2 .

We write the flappers in an alternative (to 4.1) form

$$
u_{m}(x)=2 \cos ^{-1}\left[n s\left(x+i \omega_{2}, k\right)\right]
$$

and exploit the relationship

$$
n s^{2}\left(x+i \omega_{2}\right)=\wp\left(x+i \omega_{2}\right)-e_{2}
$$

where $n s$ is the Jacobi elliptic function, $\wp$ is the Weierstrass $p$-function and the real period of $\wp$ is $1 / 2$ that of $n s$. We denote the operator $\bar{A}$ as

$$
\bar{A}=\left(\begin{array}{cc}
0 & 1 \\
Q+\delta^{2} / 2 & 0
\end{array}\right),
$$

where

$$
\begin{aligned}
Q & =\partial_{x}^{2}-\cos (u)=\partial_{x}^{2}+1-2 \cos ^{2}(u / 2) \\
& =\partial_{x}^{2}-2 \wp\left(x+i \omega_{2}\right)+\left(1+2 e_{2}\right) .
\end{aligned}
$$

This operator is a constant translate of the (negative) Lamé operator, see Magnus and Wrinkler [MW],

$$
\partial_{x}^{2}-2 \wp\left(x+i \omega_{2}\right)
$$

The periodic spectrum (period $L$ ) of $\bar{A}$ corresponds to the periodic and anti-periodic (period $L / 2$ ) spectrum of the Lamé operator which is

$$
-\mu_{1}=e_{1},-\mu_{2}=e_{3},-\mu_{3}=e_{2}
$$

with the corresponding eigenfunctions

$$
\begin{aligned}
& i c s\left(x+i \omega_{2}\right)=i\left(\wp-e_{1}\right)^{1 / 2} \\
& i d s\left(x+i \omega_{2}\right)=i\left(\wp-e_{3}\right)^{1 / 2} \\
& n s\left(x+i \omega_{2}\right)=\left(\wp-e_{2}\right)^{1 / 2} .
\end{aligned}
$$

These are Jacobi elliptic functions and we have followed the convention, $e_{1} \geq e_{3} \geq e_{2}$, in du Val [PV] in labeling the stationary values $e_{j}(j=1,2,3)$. The remaining spectrum of the Lamé operator is negative and double. The corresponding first three eigenvalues of $Q$ are

$$
\begin{aligned}
& e_{1}+2 e_{2}+1=e_{1}-e_{3}>0 \\
& e_{3}+2 e_{2}+1=0 \\
& e_{2}+2 e_{2}+1=e_{2}-e_{3}<0
\end{aligned}
$$

using

$$
e_{1}+e_{2}+e_{3}=0 \text { and } e_{1}-e_{2}=1 .
$$

The last identity is a permissible normalization. Then the identity 4.2 produces the eigenvalues of $A$, in Case 3. This was for $m=n$, where the formulae for the eigenvalues $e_{1}-e_{3}$ and $e_{2}-e_{3}$ use the identities

$$
k=L / 2 \pi n-1, \quad K=4 k /\left(1+k^{2}\right), \quad \text { and } \quad k^{2}=\frac{e_{3}-e_{2}}{e_{1}-e_{2}},
$$

where $K$ is the modulus of the $\wp$ function (and $n s$ ), $k$ is the modulus of $s n$ and the identity relating $k$ and $K$ is obtained from the energy, see the proof of Proposition 4.1, $K^{2}=h / 2$. For $m<n$, the homogeneity of the Weierstrass $p$-function and $Q$ produces the multiplicative factor $(m / n)^{2}$. 
Consider the sine-Gordon system linearized about the stationary solutions in Proposition 4.1,

$$
w_{t}=A_{k} w+\varepsilon f
$$

where $f=(0, f(x, t))$ and $k=1,2,3$.

The driving used in physical experiments is simply $f=\cos (\omega t)$. The analysis is similar for a general smooth function $f(x, t)$ but to make things concise we will assume here that $f$ has the simple time dependence $f(x, t)=f(x) \cos (\omega t)$.

Proposition 4.2. Let $f(x, t)=f(x) \cos (\omega t), T=2 \pi / \omega$, be $T$-periodic in $t$. Then there exist (linear) periodic solutions of 4.3 of the form,

(1) The flat $(u, v)=(0,0)+\underline{w}_{1}(x, t)$,

(2) The straight-up, $(u, v)=(\pi, 0)+\underline{w}_{2}(x, t)$ and

(3) $n=[L / 2 \pi]$, flappers, $\left(u_{m}(x), 0\right)+\underline{w}_{3}^{m}(x, t), m \leq n$.

If the non-resonance conditions

(1) $\omega \neq\left(1+4 \pi^{2} j^{2} / L^{2}\right)^{1 / 2}, j \in \mathbb{Z}^{+} \cup\{0\}$,

(2) $\omega \neq\left(4 \pi^{2} j^{2} / L^{2}-1\right)^{1 / 2}, j>n$,

(3) $\omega \neq(m / n)\left(e_{3}-e_{2}\right)^{1 / 2},(m / n)\left[\mu_{j}-\left(1+2 e_{2}\right)\right]^{1 / 2}, j \geq 4$,

where the $\mu_{j}$ 's are the eigenvalues of the Lamé operator in Lemma 4.1, hold respectively, then

$$
\underline{w}_{k}=\mathscr{O}(\varepsilon), \quad k=1,2,3,
$$

otherwise

$$
\underline{w}_{k}=\mathscr{O}(\varepsilon / \delta) .
$$

Proof. We write the forcing function in the form

$$
f(x, t)=\left(\sum_{k=0}^{\infty} a_{k} c_{k}(2 \pi x / L)+b_{k} s_{k}(2 \pi x / L)\right) \cos (\omega t),
$$

where $c_{k}, s_{k}$ are the usual Fourier components for Case 1 and 2, but the eigenfunctions of the Lamé operator $-d^{2} / d x^{2}+2 \wp\left(x+\omega_{2}\right)$, in Case 3. We substitute

$$
\begin{aligned}
& u(x, t)=\varepsilon \sum_{k=0}^{\infty} d_{k}(t) c_{k}(2 \pi x / L)+e_{k}(t) s_{k}(2 \pi x / L), \\
& v(x, t)=\partial u(x, t) / \partial t
\end{aligned}
$$

into the linearized equations 4.3 and choose the initial conditions so as to get the solution

$$
u(x, t)=\varepsilon \sum_{k=0}^{\infty}\left[a_{k} c_{k}(2 \pi x / L)+b_{k} s_{k}(2 \pi x / L)\right] A_{k} \cos \left(\omega t-\alpha_{k}\right),
$$

where

$$
\alpha_{k}=\tan ^{-1}\left(\omega \delta /\left(\beta_{k}^{2}-\omega^{2}\right)\right), \quad A_{k}=\left(\left(\beta_{k}^{2}-\omega^{2}\right)+\delta^{2} \omega^{2}\right)^{1 / 2}
$$

and

$$
\beta_{k}=\left(4 \pi^{2} k^{2} / L^{2}+1\right)^{1 / 2}, \quad\left(4 \pi^{2} k^{2} / L^{2}-1\right)^{1 / 2}, \quad(m / n)\left(\mu_{k}-\left(1+2 e_{2}\right)\right)^{1 / 2}
$$

respectively. If $\beta_{k}^{2} \neq \omega^{2}$ for any $k$, then $A_{k}=\mathcal{Q}(1)$, for all $k$, otherwise the presence of damping gives $A_{k}=1 / \omega \delta$, for some $k$. 
In the case of the flappers the existence of $u$ requires that the forcing function is perpendicular to the eigenfunction

$$
c_{2}=i d s\left(n\left(x+x_{1}+i \omega_{2}\right)\right)
$$

of the operator linearized about the flapper. This means that the only flapper, out of the circle, that survives, is the one which is completely out of (spatial) phase with the driving. Then we must add $a c_{2}(x)$ to the solution $u(x, t)$, where $a$ is a constant to be determined by the initial conditions. The other flappers (for $1 \leq m \leq n$ ) are similar.

Now consider the Poincaré maps associated to the time periodic equations 4.3. The spectra of these maps are easily computed.

Lemma 4.2. The spectra of the linear Poincare maps associated with the linearized equations 4.3 are

$$
\sigma\left(e^{A T}\right)=\left\{e^{\lambda_{\jmath}^{(k)} T}\right\} \quad(k=1,2,3),
$$

where the $\lambda_{j}^{k}$ 's are the eigenvalues of the three cases in Lemma 4.1.

Proof. In each of the three cases the linearized operator $A$ can be written as a function of an essentially self-adjoint operator $Q=\partial_{x}^{2}-\cos (u)$, on $\mathscr{D}(W) \subset H^{1} \times L^{2}$, with periodic boundary conditions

$$
A=\Gamma([\sqrt{Q}-(\delta / 2)] I) \Gamma^{-1},
$$

by (4.2) where $I$ is the two-dimensional identity matrix. In other words there exists a similarity transformation $\Lambda$ so that

$$
\bar{A}=\Lambda \sqrt{Q} I \Lambda^{-1}, \quad \Gamma=\Delta \Lambda,
$$

but then (4.4) follows by the spectral theorem.

We can now spell out the stability of the periodic orbits in Proposition 4.2.

Proposition 4.3. (1) The flat linear periodic orbit

$$
(0,0)+\underline{w}_{1}(x, t)
$$

is stable.

(2) The straight-up linear periodic orbit

$$
(\pi, 0)+\underline{w}_{2}(x, t)
$$

is unstable. The corresponding fixed point of the linear Poincare map $e^{A_{2} T}$ has unstable $E^{u}$ and stable $E^{s}$ subspaces, such that $\operatorname{dim} E^{u}=n+1=[L / 2 \pi]+1=$ $\operatorname{codim} E^{s}$,

$$
E^{u} \times E^{s}=H^{1} \times L^{2}[0, L] .
$$

(3) The flapper linear periodic orbits

$$
\left(u^{m}\left(x+x_{m}\right), 0\right)=\underline{w}_{3}^{m}(x, t), \quad 1 \leq m \leq n,
$$

are all unstable. The corresponding fixed points of the linear Poincaré map $e^{A_{3} T}$ have an unstable subspace $E_{m}^{u}$, a center subspace $E_{m}^{c}$ and a stable subspace $E_{m}^{s}$ such that

$$
\begin{gathered}
\operatorname{dim} E_{m}^{u}=1, \quad \operatorname{dim} E_{m}^{c}=1, \quad \operatorname{codim} E_{m}^{s}=2, \\
E_{m}^{u} \times E_{m}^{c} \times E_{m}^{s}=H^{1} \times L^{2}[0, L], \quad 1 \leq m \leq n .
\end{gathered}
$$


Proof. We apply Lemmas 4.1 and 4.2. They show that $e^{A_{1} T}$ has only eigenvalues strictly inside and in the distance $1-e^{-\delta / 2}$ from the unit circle, $e^{A_{2} T}$ has $n+1$ positive real eigenvalues outside the unit circle, the rest are inside and at least in the same distance as above from it, and that $e^{A_{3}^{m} T}$ has one positive real eigenvalue outside the unit circle, 1 is a simple eigenvalue and the rest lie inside the unit disk, and at least in the distance $1-e^{-\delta / 2}$ from it.

We can now prove that the flat and the straight-up stationary solutions perturb into nonlinear periodic orbits under the influence of the driving. The existence of the periodic orbits stemming from the flappers is a bit more complicated due to the eigenvalue 1 of the linear Poincaré map $e^{A_{3} T}$ in Lemma 4.2. It represents a marginal direction that will cause a bifurcation of each flapper.

Theorem 4.1. (1) There exists a flat (nonlinear) time-periodic solution

$$
(0,0)+w_{1}(x, t)
$$

of the damped and driven sine-Gordon equation in $H^{1} \times L^{2}[0, L]$.

(2) If $L \neq 2 \pi j, j \in \mathbb{Z}^{+}$, then there exists a straight-up (nonlinear) time-periodic solution

$$
(\pi, 0)+w_{2}(x, t)
$$

of the damped and driven sine-Gordon equation in $H^{1} \times L^{2}[0, L]$.

Proof. We consider the operators

$$
A_{\frac{1}{2}}=\left(\begin{array}{cc}
\partial_{t} & -1 \\
-\partial_{x}^{2} \pm 1 & \partial_{t}+\delta
\end{array}\right)
$$

on $\mathscr{D}(W) \subset \mathscr{H}$ where $\mathscr{H}=H^{1} \times L^{2}\left(S^{1} \times S^{1}\right)$, i.e., we look for solutions periodic in both $x$ and $t$, and $\mathscr{D}(W)$ is the domain of the wave operator in $L^{2} \times L^{2}\left(S^{1} \times S^{1}\right)$. The damped and driven sine-Gordon equation can be written in the form

$$
A(u, v)= \pm(0, u-\sin (u))+\varepsilon f,
$$

where $f=(0, f)$ and we have changed the coordinates $u \rightarrow u+\pi$ in the case of the straight-up $\left(A_{2},-\right)$. The spectra of these operators on $\mathscr{H}$ are

$$
\begin{aligned}
& \sigma\left(A_{1}\right)=\left\{-\delta / 2+\left[\frac{2 \pi}{T} k \pm\left(1-\frac{\delta^{2}}{4}+\frac{4 \pi^{2} j^{2}}{L^{2}}\right)^{1 / 2}\right] i\right\}, \quad k, j \in \mathbb{Z}, \\
& \sigma\left(A_{2}\right)=\left\{-\delta / 2+\frac{2 \pi k}{T} i \pm\left(1+\frac{\delta^{2}}{4}+\frac{4 \pi^{2} j^{2}}{L^{2}}\right)^{1 / 2}\right\},
\end{aligned}
$$

by Lemma 4.1. Proposition 4.2 says that there exist periodic solutions of the linear equations

$$
A_{1}(\underline{u}, \underline{v})=\varepsilon f
$$

and if we subtract this equation from the one above, we get

$$
A_{1} w= \pm\left(0, w^{1}+\underline{w}_{2}^{1}-\sin \left(w^{1}+\underline{w}_{2}^{1}\right)\right),
$$


where $w=(u, v)-(\underline{u, v})$, and the superscript 1 means the first coordinate $(u)$. The operators $A$ are invertible on $L^{2} \times L^{2}\left(S^{1} \times S^{1}\right)$, in fact

$$
\left\|A_{2}^{-1}\right\| \leq 2 / \delta
$$

by the above formulae for the spectrum. We apply the implicit function theorem to the equation

$$
w \mp A^{-1}\left(0, w^{1}+\underline{w}^{1}-\sin \left(w^{1}+\underline{w}^{1}\right)\right)=0 .
$$

The $w$ Frechét derivative is

$$
I \mp A^{-1}\left(\begin{array}{cc}
0 & 0 \\
\left.1-\cos \left(w^{1}+\underline{w}^{1}\right)\right) & 0
\end{array}\right)=I, \quad \text { at } \quad w=0, \quad \varepsilon=0 .
$$

Therefore there exists a solution $w(\varepsilon)$ of 4.5 in $\mathscr{D}(W) \subset \mathscr{H}$, in a neighborhood of $\varepsilon=0$. This solution is a smooth function of $\varepsilon$.

Remark 4.2. The hypothesis $L \neq 2 \pi j$ excludes 0 as an eigenvalue of $A_{2}$. If 0 is an eigenvalue, we could expect bifurcations on a one-dimensional center manifold.

Corollary 4.1. Suppose that the driving and damping amplitudes are of the same order, $\varepsilon / \delta=Q(1)$, so that parametric resonances are not present. Then the flat (nonlinear) periodic orbit is stable. The straight-up (nonlinear) periodic orbit is unstable. It corresponds to a fixed point $p$ of the nonlinear Poincaré map $P$ and with the assumption (2), in Theorem 4.1, $p$ has an unstable $W^{u}$ and a stable manifold $W^{s}$ so that

$$
\operatorname{dim} W^{u}=n+1, \quad \operatorname{codim} W^{s}=n+1,
$$

where $n=[L / 2 \pi]$.

Proof. The periodic orbits in Theorem 4.1 correspond to fixed points $p_{1}$ and $p_{2}$ respectively of the Poincare map of the damped and driven sine-Gordon equation. The stability of the periodic orbits is determined by the spectrum of $D P(p)$ the Poincare map linearized about the fixed points. We linearize the vector field about the periodic orbits and do the infinite dimensional Floquet Theory of the resulting time-periodic linear system to determine the spectrum $\sigma(D P)$.

The damped and driven sine-Gordon vector field linearized about the periodic orbits $(0,0)+w_{1}$, and $(\pi, 0)+w_{2}$ is

$$
w_{t}=\left(\begin{array}{cc}
0 & 1 \\
\partial_{x}^{2} \mp \cos \left(w^{1}(x, t)\right) & -\delta
\end{array}\right) w
$$

where $w=\left(w^{1}, w^{2}\right)$. We expand cosine to get the scalar PDE

$$
u_{t t}+\delta u_{t}+\left(1-\left(w^{1}(x, t)\right)^{2} / 2\right) u-\partial_{x}^{2} u=\mathscr{O}\left(\varepsilon^{4}\right) .
$$

We assume, for simplicity, that the driving function is such that the periodic orbit can be written as

$$
w^{1}(x, t)=\varepsilon f(x) \cos (\omega t-\alpha)+\mathscr{Q}(\varepsilon)^{2},
$$

with $f(x)$ an even function. Then we let

$$
u=\sum_{k=0}^{\infty} a_{k}(t) \cos \left(\frac{2 \pi k x}{L}\right)
$$


and get the equations

$$
\ddot{a}_{k}+\delta \dot{a}_{k}+\left( \pm 1+\frac{4 \pi^{2} k^{2}}{L^{2}}\right) a_{k}-\frac{\varepsilon^{2}}{2} \cos ^{2}(\omega t-\alpha) \sum_{j=0}^{\infty} f_{k j} a_{j}=\mathscr{Q}\left(\varepsilon^{3}\right),
$$

for the Fourier components, where

$$
f_{k j}=\frac{2}{L} \int_{0}^{L} f^{2}(x) \cos \left(\frac{2 \pi k x}{L}\right) \cos \left(\frac{2 \pi j x}{L}\right) d x, \quad k, j \in \mathbb{Z}^{+} \cup\{0\} .
$$

If $f(x)$ is odd, we get similar equations for the sine Fourier components and in general we get a coupled system of the two, however the analysis is similar in that case.

We write the above equations as an infinite dimensional system of Mathieau's equations

$$
\ddot{a}_{k}+\delta \dot{a}_{k}+\omega_{k} a_{k}-\frac{\varepsilon^{2}}{4}[1+\cos (2 \omega t-2 \alpha)] \sum_{j=0}^{\infty} f_{k j} a_{\jmath}=\mathcal{O}^{\prime}\left(\varepsilon^{3}\right) .
$$

This system is analyzed in Birnir and Smereka [BS], or Nayfeh and Mook [NM]. The conditions for stability (and instability) are

$$
\varepsilon^{2}<\left(2 \omega_{m} \omega_{n} \Lambda_{m n}^{-1 / 2} /\left(\omega_{m}^{2}+\omega_{n}^{2}\right)\right)\left[\left(\Omega-\left(\omega_{m}+\omega_{n}\right)\right)^{2}+\frac{\delta^{2}}{4}\left(\omega_{m}^{2}+\omega_{n}^{2}\right)\right]^{1 / 2},
$$

at $\Omega=2 \omega=\omega_{m}+\omega_{n}$, where

$$
\Lambda_{m n}=f_{m n} f_{n m} / \omega_{m} \omega_{n} .
$$

At $\Omega=2 \omega=2 \omega_{m}$, this becomes

$$
\varepsilon^{2}<\left(\omega_{m} / f_{m m}\right)\left[\left(\Omega-2 \omega_{m}\right)^{2}+\delta^{2}\right]^{1 / 2} .
$$

The above are secondary parametric resonances, whereas at a primary parametric resonance $\Omega=2 \omega=\omega_{k}$, we have the condition

$$
\varepsilon<c \delta
$$

for stability, where $c<2$ is a constant. All the higher order resonances are weaker.

Now with the above conditions satisfied, the spectra

$$
\sigma\left(D P\left(p_{k}\right)\right) \text { and } \sigma\left(e^{A_{k} T}\right), \quad k=1 \text { or } 2,
$$

are qualitatively the same. The latter spectra is spelled out in Lemma 4.2. Then the existence of the stable and unstable manifolds of $p_{2}$ are given by the stable-and unstable manifold theorem for maps, see Hartman $[\mathrm{H}]$.

We apply the center-manifold theorem, see Bates and Jones [BJ] and Carr [C], to show that each flapper can give three periodic orbits because a pitchfork bifurcation takes place as one eigenvalue of the Poincaré map crosses the unit circle at 1 , under the influence of the nonlinearities and the driving.

The center-manifold computations for the flappers and carried out in the Appendix which contains the proofs of Proposition 4.4 and 4.5.

We now restrict the forcing further to get a form exhibiting the bifurcations of the physical forcing $\cos (\omega t-\phi)$. 
Proposition 4.4. Suppose the forcing function has the form

$$
f(x, t)=f(x) \cos (\omega t-\phi),
$$

where $f(x)$ is even. Then the damped and driven sine-Gordon equation at a flapper can be split into two ODE's,

$$
\begin{aligned}
\dot{a}= & \alpha a^{3}+\beta a^{2} \varepsilon \cos (\omega t-\phi)+\gamma a \varepsilon^{2} \cos ^{2}(\omega t-\phi) \\
& +\mu \varepsilon^{3} \cos ^{3}(\omega t-\phi)+\mathscr{\odot}(a, b, \varepsilon)^{4}, \\
\dot{b}= & -\delta b+\mathscr{\odot}(a, b, \varepsilon)^{4},
\end{aligned}
$$

and $a \mathrm{PDE}$

$$
\frac{\partial y}{\partial t}=A\left(u_{m}\right) y+\left(\begin{array}{c}
0 \\
-2 i d s^{\prime}(a i d s+\varepsilon f \cos (\omega t-\phi))^{2}
\end{array}\right)+\mathscr{O}(a, b, \varepsilon)^{3},
$$

where

$$
A\left(u_{m}\right)=\left(\begin{array}{cc}
0 & 1 \\
-\partial_{x}^{2}+\cos \left(u_{m}(x)\right) & -\delta
\end{array}\right),
$$

$d s$ is the Jacobi elliptic function, $d s^{\prime}$ its derivative, and the coefficients $\alpha, \beta, \gamma, \mu$ are given in Table A.1.

Proof. See the Appendix.

Proposition 4.5. The time-periodic solutions, $T=2 \pi / \omega$, of the ODE

$$
\dot{a}=\alpha a^{3}+\beta a^{2} \varepsilon \cos (\omega t-\phi)+\gamma a \varepsilon^{2} \cos ^{2}(\omega t-\phi)+\mu \varepsilon^{3} \cos ^{3}(\omega t-\phi)+\mathscr{Q}(a, 0, \varepsilon)^{4},
$$

bifurcate by a pitchfork bifurcation into three branches as $0 \leq \varepsilon$ increase, if $\gamma(f)<0$. Two of these branches are stable, one is unstable. If $\gamma(f)>0$, there is only one unstable branch.

Proof. See the Appendix

Now we can prove that the flappers undergo a pitchfork bifurcation, for $f(x)=1$.

Theorem 4.2. For every $m, 1 \leq m \leq n=[L / 2 \pi]$, there exist three (nonlinear) time-periodic flapper solutions

$$
\left(u_{m}(x), 0\right)+w_{3}^{[k]}(x, t, m), \quad k=1,2,3,
$$

of the damped and driven sine-Gordon equation in $H^{1} \times L^{2}[0, L]$, if $\gamma(f)<0$, in Table A.1. These are all unstable but two correspond to a fixed point $p_{2+j m}$ of the nonlinear Poincaré map $P$ with unstable $W_{\jmath}^{u}$ and stable manifolds $W_{\jmath}^{s}$,

$$
\operatorname{dim} W_{\jmath}^{u}=1, \quad \operatorname{codim} W_{j}^{s}=1, \quad j=1,3,
$$

whereas the third corresponds to the fixed point $p_{2+2 m}$ with

$$
\operatorname{dim} W_{2}^{u}=2, \quad \operatorname{codim} W_{2}^{s}=2 .
$$

If $\gamma(f)>0$, only one flapper exists for each $m \leq n$, with unstable and stable manifolds of dimension and codimension 1 , respectively. 
Proof. We apply the Lyapunov-Schmidt method, see Chow and Hale $[\mathrm{CH}]$, to the equation

$$
\left(\begin{array}{cc}
\partial_{t} & -1 \\
\partial_{x}^{2}+\cos \left(u_{m}\right) & \partial_{t}+\delta
\end{array}\right) z=N\left(z, \underline{w}_{3}\right),
$$

which was derived in the proof of Proposition 4.4. Recall that $z=w-\underline{w}$ and

$$
N\left(z, \underline{w}_{3}\right)=\left(0-\left[\sin \left(z_{1}-\left(u_{m}+\varepsilon \underline{u}_{3}\right)\right)-\cos \left(u_{m}\right) z_{1}-\varepsilon \cos \left(u_{m}\right) \underline{u}_{3}\right]\right) .
$$

The linear operator acts on the space $\mathscr{H}=H^{1} \times L^{2}\left(S^{1} \times S^{1}\right)$ and is defined on $\mathscr{D}(W) \subset \mathscr{H}$, where $\mathscr{D}(W)$ is the domain of the wave operator. By Lemma 4.1 the operator

$$
A_{m}=\left(\begin{array}{cc}
\partial_{t} & -1 \\
-\partial_{x}^{2}+\cos \left(u_{m}\right) & \partial_{t}+\delta
\end{array}\right)
$$

has the eigenvectors $(u, v)=\left(1, \lambda_{j}^{(3)}\right) f_{j} e^{2 \pi i k t / T}$, where the $\lambda_{j}^{(3)}$ 's are the eigenvalues in Lemma 4.1 and the $f_{\jmath}$ 's are the eigenfunctions of the Lamé operator $-\partial_{x}^{2}+2 \wp$ with eigenvalues $(m / n)^{2} \mu_{j}$. The corresponding eigenvalues of $A_{m}$ are

$$
\begin{aligned}
\eta_{k \jmath} & =2 \pi i k / T-\lambda_{j} \\
& =-\delta / 2+\left[2 \pi k / T \pm\left((m / n)^{2}\left(\mu_{\jmath}-\left(1+2 e_{2}\right)\right)-\delta^{2} / 4\right)^{1 / 2}\right] i, \quad j, k \in \mathbb{Z} .
\end{aligned}
$$

Only one of these eigenvalues vanishes

$$
\eta_{02}=0
$$

and the projection onto the corresponding dual vector is

$$
\Pi(u, v)=\int_{0}^{T} \Pi_{1}(u, v) d t
$$

where $\Pi_{1}$ is the projection in the proof of Proposition 4.4. Now we split Eq. (4.6) into

$$
A_{m} y=(I-\Pi) N(x+y, w)
$$

and

$$
\Pi N(x+y, w)=0 .
$$

Here $z=x+y$, where

$$
x=a\left(\begin{array}{l}
1 \\
0
\end{array}\right) i d s
$$

lies in kernel $A_{m}$ and $y \in \operatorname{ker} A_{m}^{\perp}$. The latter equation (4.7) is just the bifurcation equation in Proposition 4.4, it gave us three branches of periodic solutions in the kernel, by Proposition 4.5. Now the operator $A_{m}$ is invertible on $\operatorname{ker} A_{m}^{\perp}$, the subspace orthogonal to the kernel. By the formula for the eigenvalues $\eta_{k j}$ above we have

$$
\left\|A_{m}^{-1}\right\| \leq 2 / \delta \text { on } \operatorname{ker} A_{m}^{\perp} .
$$

We apply the implicit function theorem to the equation

$$
y-A_{m}^{-1}(I-\Pi) N(x+y, w)=0 .
$$


It has the solution $y \equiv 0$ at the origin $x=0=y, \varepsilon=0$, recall that $w=\mathscr{O}(\varepsilon)$. Moreover, the Frechét derivative of $N$ is

$$
D_{y} N=\left(\begin{array}{cc}
0 & 0 \\
\cos \left(z_{1}-\left(u_{m}+\varepsilon \underline{u}_{3}^{1}\right)\right)-\cos \left(u_{m}\right) & 0
\end{array}\right)
$$

so that

$$
D_{y} N(0,0)=0 \quad \text { and } \quad\left\|D_{y} N\right\| \leq 2
$$

Therefore

$$
D_{y}\left(y-A_{m}^{-1}(I-\Pi) N(x+y, w)\right)=I-A_{m}^{-1}(I-\Pi) D_{y} N(x+y, w)=I \neq 0,
$$

at $x=0=y, \varepsilon=0$. By the implicit function theorem there exists a smooth solution $y(x(a), \varepsilon)$ of 4.8 in $\mathscr{H}$, for each branch of $a$.

Now recall from Theorem 3.3 the decomposition of the global attractor $\mathscr{A}$ into the basic attractor $\mathscr{B}$ and the "hyperbolic" structure $\mathscr{C}$. We summarize the components of $\mathscr{B}$ and $\mathscr{C}$ that we have so far.

Proposition 4.6. Let $p_{1}$ be the fixed point of the Poincare map of the damped and driven sine-Gordon equation corresponding to the flat periodic orbit, $p_{2}$ the fixed point corresponding to the straight-up periodic orbit and $p_{2+m \jmath}$, the fixed points corresponding to the flapper periodic orbits. Then

$$
p_{1} \in \mathscr{B} \quad \text { and } \quad p_{2}, p_{2+m j} \in \mathscr{C}, \quad 1 \leq m \leq n, \quad 1 \leq j \leq 3 .
$$

Moreover, the unstable manifolds $W^{u}\left(p_{2}\right)$ and $W^{u}\left(p_{2+m j}\right)$ belong to $\mathscr{C}$.

Proof. $p_{1}$ is stable by Corollary 4.1. Thus $p_{1}$ attracts a full neighborhood $U$ of $p_{1}$ in $H^{1} \times L^{2}[0, L]$ (with periodic boundary conditions). However, for $u$ small the metric on $\overline{\mathscr{H}}=\bar{H}^{1} \times \bar{L}^{2} \times S^{1} \times \mathbb{R}$ is equivalent to the norm on $H^{1} \times L^{2}$, because $1-\cos (u)=u^{2} / 2+\mathcal{Q}\left(u^{4}\right)$. Now let hat denote the mean and bar mean-free as in Sect. 2 and 3. Then $p_{1}=\left(\bar{p}_{1}, \widehat{p}_{1}\right)$ and $U=\bar{U} \times \widehat{U}$, where $\bar{p}_{1}$ and $\bar{U}$ lie in $\bar{H}^{1} \times \bar{L}^{2}$ and $\widehat{p}_{1}$ and $\widehat{U}$ lie in $S^{1} \times \mathbb{R}$. Moreover, $\mathscr{A}, \mathscr{B}$ and $\mathscr{C}$ split as in the proof of Corollary 3.1; if $m \geq 2 c(\overline{\mathscr{C}})+1$ and $\Pi$ any projection onto $\mathbb{R}^{m}$, then by the open mapping theorem $\Pi(U)$ is open in $\mathbb{R}^{m}$. This means that the Lebesgue measure in $\mathbb{R}^{m}$,

$$
\bar{\mu}(\Pi(\bar{U}))>0, \quad \text { for all } \Pi \in V,
$$

where $V$ is the residual set of projections which are one to one on $\overline{\mathscr{b}}$, see Theorem 3.3. Consequently,

$$
\bar{p}_{1} \in \bigcap_{\Pi \in V} \Pi^{-1}\left(B_{\Pi}\right)=\overline{\mathscr{B}}
$$

Moreover, $\widehat{\mu}(\widehat{U})>0$, where $\widehat{\mu}$ is Lebesgue measure in $S^{1} \times \mathbb{R}$, so $\widehat{p} \in \widehat{\mathscr{B}}$ and

$$
p_{1}=\left(\bar{p}_{1}, \widehat{p}_{2}\right) \in \overline{\mathscr{B}} \times \widehat{\mathscr{B}}=\mathscr{B} \text {. }
$$

The straight-up fixed point $p_{2}$ is unstable by Corollary 4.1 and the flappers $p_{2+\jmath m}$ 's are all unstable by Theorem 4.2. All of these points belong to the global attractor - A because they have a stable manifold of codimension $\leq 2$. However, all of these points also have an unstable manifold $W^{u}$ of dimension $\geq 1$, see Corollary 4.1 and Theorem 4.2. Now let $p=(\bar{p}, \widehat{p})$ be one of these points and $\bar{W}^{s}$ and $\bar{W}^{u}$ its stable 
and unstable manifold respectively in $\bar{H}^{1} \times \bar{L}^{2}$. If $\operatorname{dim} \bar{W}^{u} \geq 1$, then it is easy to see, since $\bar{W}^{s}$ is a manifold, that for a sufficiently small neighborhood $\bar{U}$ of $\bar{p}$ in $\bar{W}^{s}$, we can find a $\Pi \in V$ such that the Lebesgue measure $\bar{\mu}(\Pi(U))=0$, in $\mathbb{R}^{m}$, $m \geq 2 c(\overline{\mathscr{A}})+1$. If on the other hand $\operatorname{dim} \bar{W}^{u}=0$, then $\operatorname{dim} \widehat{W}^{u}=1$, because $\operatorname{dim} \widehat{W}^{u}=\operatorname{dim} \widehat{W}^{s}$, by Corollary 4.1 and Theorem 4.2. Consequently, $\widehat{\mu}(\widehat{U})=0$, for any $\widehat{U} \subset \widehat{W}^{s}$. In either case the product measure in $\mathbb{R}^{n}, n=m+2 \geq 2 c(\mathscr{b})+1$, is zero

$$
\mu(\Pi(\bar{U}) \times \widehat{U})=\bar{\mu}(\Pi(\bar{U})) \widehat{\mu}(\widehat{U}))=0
$$

so $p=(\bar{p}, \hat{p}) \in \overline{\mathscr{C}} \times \hat{\mathscr{C}}=\mathscr{C}$. We conclude that

$$
p_{2}, p_{2+j m} \in \mathscr{C}, \quad 1 \leq m \leq n, \quad 1 \leq j \leq 3 .
$$

The stable and unstable manifolds of $p_{2}$ and $p_{2+j m}$ have global existence since the perturbed sine-Gordon flow and consequently its Poincaré map is reversible and the nonlinearity is Lipschitz, see Bates and Jones [BJ]. By Remark 3.2, the $A$-attractor is connected, therefore its hyperbolic components must be connected by their unstable manifolds which also belong to $\mathscr{A}$. The $B$-attractor contains no unstable manifolds since unstable manifolds do not attract a full neighborhood up to projected measure zero. Therefore, $\mathscr{C}$ the complement of $\mathscr{B}$ in $\mathscr{C}$ must contain the unstable manifolds.

Unstable components of $\mathscr{A}$ and their unstable manifolds can conceivably form a closed cycle, since such heteroclinic connections are structurally unstable one expects these only at isolated parameter values. In the presence of symmetries heteroclinic connections may be structurally stable. However, their existence requires strong forcing because even small damping accumulates along the connection and the orbit does not "make it" up to the same energy level again without strong driving. The parameter values (of $\varepsilon$ ) that we are considering do not allow for the stabilization of any heteroclinic cycles. However, for much larger driving amplitudes such a nonperturbative stabilization takes place and this is explored in Armbruster, Birnir and Buys $[\mathrm{ABB}]$.

The above analytical results hold for $\varepsilon$ and $\delta$ small enough and a class of forcing functions including the physically relevant $f=\cos (\omega t)$. We have not used any numerical information except to guide the analysis, but one can explore how large $\varepsilon$ and $\delta$ can become so that the above results persist. Moreover, one can find all the components of $\mathscr{B}$ (this is easy because they are attracting) and at least some of the components of $\mathscr{C}$ (this is harder but possible because of the presence of the stable manifolds).

Remark 4.3. It is easy to see that Proposition 4.6 gives a complete description of the global attractor for sufficiently small damping and driving parameters. The reason is that for $\delta$ fixed and $\varepsilon$ sufficiently small the energy $\mathscr{E}$ in Sect. 2 is a Lyapunov function for the damped and driven sine-Gordon equation and we can use the results of Babin and Vishik [BV] to show that

$$
\mathscr{B}=p_{1}, \quad \mathscr{C}=p_{2} \cup W_{2}^{u} \cup\left\{p_{3+m \jmath} \cup W_{3+m \jmath}^{u}\right\} \quad 1 \leq m \leq n, \quad 1 \leq j \leq 3,
$$

and

$$
\mathscr{b}=\mathscr{B} \cup \mathscr{C} \text {. }
$$

We must allow for the fact that the flappers are not hyperbolic but this is easily taken care of using the center-manifold in the Appendix. Numerically we can show 
that this is a complete description of the attractor below the line $\Gamma$ in the $\delta-\varepsilon$ plane on Fig. 5.1. It is remarkable that in this region closest to the completely integrable unperturbed sine-Gordon equation, none of the integrable tori survive in the long-time asymptotics.

\section{The $B$-Attractor and the Hyperbolic Structure}

In Sect. 4, we described one component of the $B$-attractor, the stable flat, and $3 n+1$ components of the "hyperbolic" structure, the straight-up and $3 n(n=[L / 2 \pi])$ flappers.

In this section we investigate other components of the $\mathscr{B}$-attractor, that exist above the curve $\Gamma$ in Fig. 5.1. These are the most interesting components of $\mathscr{B}$ consisting of spatially localized breathers that exhibit very complex bifurcations, see Grauer and Birnir [GB], as $\varepsilon$ and $\delta$ vary.

The breather on the interval can be expressed, see Date [D], as

$$
u(x, t)=-i \log \left[\frac{\Theta(w(\mathscr{P})+c)}{\Theta(w(\mathscr{P})+\overline{1 / 2}+c)}\right],
$$

where $\Theta$ is the theta function associated to a Riemann surface $R$ of genus 2, see for example Birnir [B1] and Siegel [S] for more information on such surfaces and theta functions. $w(\mathscr{P})=\int_{\mathscr{D}}^{\mathscr{P}}\left[\omega_{\imath}\right](i=1,2,3,4)$ is a four vector whose entries are a basis of differentials of the first kind on $R, \mathscr{P}$ is a divisor consisting of points on $R, c$ is the Riemann constant and $\overline{1 / 2}$ is the four vector with entries $1 / 2$. In general $u$ may have irremovable singularities, see Birnir [B2,3], but here $u$ is smooth (in fact analytic) due to the symmetry of $R$, see Date [D], that also makes $u$ real. $w(\mathscr{P})$ constitutes the Jacobi map, see Siegel [S], from the four fold symmetric product of $R$ onto $\mathscr{J}=\mathbb{C}^{4} / \Lambda$, the Jacobi variety of $R$. This is a four torus, $\Lambda$ is the period lattice of $R$, which constitutes an action-angle manifold of the unperturbed sineGordon equation. On $\mathscr{J}$, translation and the sine-Gordon flow proceed along straight lines with constant speed, see McKean [McK] and Forest and McLaughlin [FM], and $w(\mathscr{P})=x t_{1}+t t_{2}$, where $t_{1}$ and $t_{2}$ (the tangent vectors of translation and the sine-Gordon flow respectively) are constant vectors. This gives us the formula

$$
u(x, t)=-i \log \frac{\Theta\left(x t_{1}+t t_{2}+c\right)}{\Theta\left(x t_{1}+t t_{2}+\overline{1 / 2}+c\right)}
$$

for the breather.

This function has four parameters $x_{0}, t_{0}, t_{1}(\omega), t_{2}(v)$, parametrizing spatial and temporal translation, amplitude scaling and the Lorentz boost. We restrict ourselves to the subspace of even functions

$$
Y=\left\{w \in H^{1} \times L^{2}[0, L] \mid w(x)=w(-x)\right\},
$$

for simplicity. This amount to posing the initial value problem for the PDE with even initial data. Then spatial translation and the Lorentz boost are eliminated and we are left with only two parameters $t_{0}$ and $\omega$. We will prove the existence of the breather solutions of the damped and driven sine-Gordon equation, but first we need a technical lemma. 
Lemma 5.1. The kernel of the sine-Gordon equation

$$
\left(\begin{array}{cc}
\partial_{t} & -1 \\
-\partial_{x}^{2}+\cos \left(u_{B}\right) & \partial_{t}
\end{array}\right) w=0
$$

linearized about the breather, in $H^{1} \times L^{2}[0, L] \cap Y$, is spanned by $\partial_{t}(u, v)_{B}, \partial_{\omega}(u, v)_{B}$ and the functions $\left(\psi_{n}^{ \pm}, \partial_{t} \psi_{n}^{ \pm}\right)$, where

$$
\psi_{n}^{ \pm}=\exp \left[i\left(\frac{2 \pi n x}{L} \pm \omega_{n} t\right)\right] \times \frac{\Theta\left(x t_{1}+t t_{2}+c_{n}\right)}{\Theta\left(x t_{1}+t t_{2}+\frac{1}{2}+c_{n}\right)}
$$

$\overline{1 / 2}$ is the four-vector with entries $1 / 2$, the $c_{n}$ 's are constants and $\omega_{n}=\left(4 \pi^{2} n^{2}+\right.$ $1)^{1 / 2} / L, n \in \mathbb{Z}$.

Proof. It is a general fact of inverse spectral theory that the product of the eigenfunctions of the linear spectral problem associated to the PDE span (here $H^{1} \times L^{2}[0, L]$ with periodic boundary conditions), and satisfy the variational equation of the PDE. The reason is that the variation of the solution to the PDE can be written in terms of the product of eigenfunctions, see McKean [McK] and McLaughlin and Scott [MS]. Now

$$
\psi_{n}^{ \pm}=f_{1}^{ \pm} f_{2}^{ \pm}
$$

where $\left(f_{1}^{ \pm}, f_{2}^{ \pm}\right)$is the solution to the linear spectral equation. We write the latter as an exponential function times a quotient of theta-functions, see Date [D], and simplify the latter. Evenness in $x$, is imposed by freezing $x_{0}=0$ or $L / 2$.

We will need a bound on the damped Poincaré map to prove the existence of the spatially symmetric breathers. First we scale $t$ by $\omega$ to make the $t$-period $2 \pi$.

Lemma 5.2. The Poincaré map of the damped sine-Gordon equation linearized about the breather

$$
\left(\begin{array}{cc}
\omega \partial_{t} & -1 \\
-\partial_{x}^{2}+\cos \left(u_{B}\right) & \omega \partial_{t}-\delta
\end{array}\right) w=0
$$

is $D P=1$, on the one-dimensional subspace of $H^{1} \times L^{2}[0, L] \cap Y$ spanned by $\partial_{t}(u, v)_{B}+\delta h_{0}$; is $D P=\exp (-\delta T)$ on the one-dimensional subspace spanned by $\partial_{\omega}(u, v)_{B}+\delta h_{1} ;$ and is

$$
D P=\exp (-\delta T)\left(\begin{array}{cc}
\exp \left(i \omega_{n}^{\prime} 2 \pi\right) & 0 \\
0 & \exp \left(-i \omega_{n}^{\prime} 2 \pi\right)
\end{array}\right)
$$

on each two dimensional subspace spanned by

$$
\left(\psi_{n}^{ \pm}, \partial_{t} \psi_{n}^{ \pm}\right)+\delta^{2} h_{n}^{ \pm}, \quad n \in \mathbb{Z}
$$

where $T=2 \pi / \omega$ and $\omega_{n}^{\prime}=\left(\omega_{n}^{2}-\delta^{2} / 4 \omega^{2}\right)^{1 / 2}$. 
Proof. We use the radiation $\psi_{n}^{ \pm}=e^{ \pm i \omega_{n} t} g_{n}^{ \pm}$, in Lemma 5.1, to compute a basis of solutions to the damped equation. The $g$ 's are periodic in $x$ and $t$ and satisfy the equation

$$
\left(-\partial_{x}^{2}+\cos \left(u_{B}\right)\right) g_{n}=\omega^{2}\left[\omega_{n}^{2} g_{n}-2 i \omega_{n} g_{n t}-g_{n t t}\right]
$$

since the $\psi$ 's satisfy the equations

$$
\left(-\partial_{x}^{2}+\cos \left(u_{B}\right)\right) \psi_{n}=-\omega^{2} \partial_{t}^{2} \psi_{n} .
$$

We look for solutions of the damped equation

$$
\left(-\partial_{x}^{2}+\cos \left(u_{B}\right)\right) \psi=-\omega^{2} \partial_{t}^{2} \psi-\delta \omega \partial_{t} \psi
$$

of the form

$$
\psi(\delta)=\sum \alpha_{k} g_{k} e^{\beta_{k} t}+\delta h^{1}+\delta^{2} h_{2}^{1},
$$

where the superscript means first component and we normalize the functions $g_{k}$, $g_{0}=\partial_{t} u_{B}$ and $g_{1}=\partial_{\omega} u_{B}$, to be of norm 1 in $\mathscr{H}=H^{1} \times L^{2}\left(S^{1} \times S^{1}\right)$ :

$$
\int_{0}^{2 \pi} \int_{0}^{L} g_{k}^{2} d x d t=1
$$

A substitution of (5.5) into (5.4) produces $\beta_{k}^{2}+\beta_{k} \delta / \omega=-\omega_{k}^{2}$ and the remainder

$$
\begin{aligned}
& \delta \alpha_{0} \omega g_{0 t}-\delta \alpha_{1} \omega g_{1 t}+2 i \omega^{2} \sum_{k \neq 0,1} \alpha_{k}\left[\left(\omega_{k}^{\prime}-\omega_{k}\right) g_{k t}\right] \\
& \quad+\left[\omega^{2} \partial_{t}^{2}+\delta \omega \partial_{t}-\partial_{x}^{2}+\cos \left(u_{B}\right)\right]\left(\delta h^{1}+\delta^{2} h_{2}^{1}\right)=0,
\end{aligned}
$$

where $\omega_{n}^{\prime}-\omega_{n}=\mathscr{Q}\left(\delta^{2}\right)$ and $h=\left(h^{1}, h^{2}\right)$. Now we let $h^{1}=\alpha_{0} h_{0}^{1}+\alpha_{1} h_{1}^{1}$, then $h_{0}^{1}$ must satisfy the equation

$$
\left[\omega^{2} \partial_{t}^{2}+\delta \omega \partial_{t}-\partial_{x}^{2}+\cos \left(u_{B}\right)\right] h_{0}^{1}=\omega g_{0 t} .
$$

The right-hand side of this equation is orthogonal to $g_{0}$ and $g_{1}$ which span the kernel of $\left[\omega^{2} \partial_{t}^{2}-\partial_{x}^{2}+\cos \left(u_{B}\right)\right]$ in $H^{1}\left(S^{1} \times S^{1}\right)$,

$$
\int_{0}^{L} \int_{0}^{2 \pi} g_{0 t} g_{0} d t d x=0=\int_{0}^{L} \int_{0}^{2 \pi} g_{0 t} g_{1} d t d x .
$$

The first orthogonality is by periodicity in $t$ and the second is obtained by differentiating

$$
\int_{0}^{2 \pi} \int_{0}^{L} g_{0}^{2} d x d t=1
$$

with respect to $\omega$.

We use that both $\left\{\psi_{n}^{ \pm}\right\}$and $\left\{\partial_{t} \psi_{n}^{ \pm}\right\}$span $H^{1}$ by Lemma 5.1 and express

$$
g_{0 t}=\sum_{k=2}^{\infty} b_{k}(t) g_{k}+c_{k}(t) g_{k t}
$$

where the Fourier coefficients $b_{k}$ and $c_{k}$ are periodic with period $2 \pi$. A relationship between the $b_{k}$ 's and $c_{k}$ 's will be established in a moment. The orthogonality above 
allows us to omit $g_{0}$ and $g_{1}$. Then let $h_{0}^{1}=\sum_{k=2}^{\infty} a_{k} g_{k}$ and substitute into the equation for $h_{0}^{1}$ using the equation for the $g_{k}$ 's above. We end up with two equations

$$
\begin{aligned}
\dot{a}_{k}+\left(\frac{\delta}{2 \omega}-i \omega k\right) a_{k} & =\frac{c_{k}}{\omega}, \\
\ddot{a}_{k}+\frac{\delta}{\omega} \dot{a}_{k}+\omega_{k}^{2} a_{k} & =\frac{b_{k}}{\omega},
\end{aligned}
$$

for the coefficients $a_{k}$. Now we expand the periodic functions of $t$

$$
c_{k}=\sum_{n=0}^{\infty} c_{k}^{n} e^{i n t}, \quad b_{k}=\sum_{n=0}^{\infty} b_{k}^{n} e^{i n t}
$$

and obtain a solution of the above equations if

$$
c_{k}^{n}=\frac{\left[i\left(n-\omega_{k}\right)+\delta / 2 \omega\right]}{\left(\omega_{k}^{2}-n^{2}\right)+i n \delta / \omega} b_{k}^{n}, \quad n=0,1, \ldots,
$$

namely,

$$
a_{k}(t)=a_{k}^{0} e^{-\left(\frac{\delta}{2 \omega}-\imath \omega_{k}^{\prime}\right) t}+q_{k}
$$

where

$$
q_{k}(t)=\sum_{n=0}^{\infty} \frac{b_{k}^{n} e^{\imath n t}}{\omega\left(\left(\omega_{k}^{2}-n^{2}\right)+i n \delta / \omega\right)}
$$

is periodic with period $2 \pi$. Thus

$$
h_{0}^{1}=\sum_{k=2}^{\infty}\left(a_{k}^{0} e^{\beta_{k} t}+q_{k}\right) g_{k} .
$$

The equations

$$
\left[\omega^{2} \partial_{t}^{2}+\delta \omega \partial_{t}-\partial_{x}^{2}+\cos \left(u_{B}\right)\right] h_{1}^{1}=-\omega g_{1 t}
$$

and

$$
\left[\omega^{2} \partial_{t}^{2}+\delta \omega \partial_{t}-\partial_{x}^{2}+\cos \left(u_{B}\right)\right] h_{2 k}^{1}=\left(\frac{\omega_{k}^{\prime}-\omega_{k}}{\delta^{2}}\right) g_{k t},
$$

where $h_{2}^{1}=\sum_{k=2}^{\infty} \alpha_{k} h_{2 k}^{1}$, are solved similarly to give

$$
h_{1}^{1}=\sum_{k=2}^{\infty}\left(d_{k}^{0} e^{\beta_{k} t}+r_{k}\right) g_{k}
$$

and

$$
h_{2 k}^{1}=\sum_{\jmath=2}^{\infty}\left(e_{\jmath}^{0} e^{\beta_{\jmath} t}+s_{\jmath}^{k}\right) g_{j}, \quad k=2, \ldots,
$$

The upshot is that we can absorb the coefficients $a_{k}^{0}, d_{k}^{0}$ and $e_{k}^{0}$ into $\alpha_{k}$ and express $\psi$ as

$$
\psi(t)=\sum_{k=0}^{\infty} \alpha_{k} e^{\beta_{k} t} g_{k}+\delta\left(\alpha_{0} h_{0}^{1}+\alpha_{1} h_{1}^{1}\right)+\delta^{2} h_{2}^{1},
$$


where the $h$ 's are periodic functions. The only thing one needs to be careful about is that the $h$ 's are $\mathscr{Q}(1 / \delta)$, but not $\mathscr{Q}(1)$ because of the resonances, $n \sim \omega_{k}, k=2, \ldots$, . The form of the Poincare map is now immediate

$$
\begin{aligned}
\psi(t+2 \pi)= & g_{0}(t)+g_{1}(t) e^{-\delta(t+2 \pi) / \omega} \\
& +\sum_{k \neq 0,1} \alpha_{k} g_{k} e^{\beta_{k}(t+2 \pi)}+\delta h^{1}(t)+\delta^{2} h_{2}^{1}(t) .
\end{aligned}
$$
in $x$ ),

Now we assume that the forcing is an even function of $x$ (to freeze translation

$$
f(x, t)=\sum_{k=1}^{\infty} a_{k}(x) \cos (k \omega t)+b_{k}(x) \sin (k \omega t)
$$

and state and prove the existence of the breathers for

$$
f(x, t)=f(x) \cos (\omega t-\phi),
$$

for simplicity. The proof is the same for the more general form of $f$ above. We scale $t$ by $\omega$ as in Lemma 5.2 to fix the space $\mathscr{H}=H^{1} \times L^{2}\left(S^{1} \times S^{1}\right)$.

Theorem 5.1. Let $f(x, t)=f(x) \cos (t-\phi / \omega), f(x)$ even, and suppose that

$$
\varepsilon \geq \delta \int_{-L / 2}^{L / 2} \int_{-\pi}^{\pi} v_{B}^{2} d t d x / \int_{-L / 2}^{L / 2} \int_{-\pi}^{\pi} f(x) \cos (t) v_{B} d t d x
$$

where

$$
v_{B}=-\frac{i d}{d t} \log \left[\frac{\Theta\left(x t_{1}+t t_{2} / \omega+c\right)}{\Theta\left(x t_{1}+t t_{2} / \omega+\overline{1}+c\right)}\right]
$$

is the derivative of the breather. Then there exist two pairs of breather solutions, one stable the other unstable, of the damped and driven sine-Gordon equation in $\mathscr{H} \cap Y$.

Proof. We write the damped and driven sine-Gordon equation in the form

$$
A_{B} w=\left(\begin{array}{c}
0 \\
-\sin (u)+\cos \left(u_{B}\right) u
\end{array}\right)+\varepsilon f
$$

where $f=(0, f(x, t)), w=(u, v)$, and $A_{B}$ is the linear operator

$$
A_{B}=\left(\begin{array}{cc}
\omega \partial_{t} & -1 \\
-\partial_{x}^{2}+\cos \left(u_{B}\right) & \omega \partial_{t}+\delta
\end{array}\right) .
$$

The breather itself satisfies the equation

$$
A_{B} w_{B}=\left(\begin{array}{c}
0 \\
-\sin \left(u_{B}\right)+\cos \left(u_{B}\right) u_{B}+\delta v_{B}
\end{array}\right) .
$$

We subtract this equation from the one above to get

$$
A_{B} z=N(z, \delta)+\varepsilon f
$$

where $z=w-w_{B}$, and

$$
N(z, \delta)=\left(0,-\sin \left(z_{1}+u_{B}\right)+\sin \left(u_{B}\right)+\cos \left(u_{B}\right) z_{1}+\delta v_{B}\right) .
$$


We will use a periodic version of the Lyapunov-Schmidt method to prove the existence of periodic solutions of the last equation. By Lemma 5.2, $A_{B}$ has a one dimensional kernel in the subspace $\mathscr{H} \cap Y$ of even function in $\mathscr{H}$. Let $z=s+y$, where $s \in \operatorname{kern} A_{B}$ and $y \in \operatorname{kern} A_{B}^{\perp}$. If $\Pi$ denotes the projection onto kern $A_{B}$, then the equation above can be written as two equations

and

$$
\begin{aligned}
& A_{B} y=(I-\Pi)(N(z, \delta)+\varepsilon f) \\
& \Pi(N(z, \delta)+\varepsilon f)=0 .
\end{aligned}
$$

First consider the second equation. It can be written

$$
\begin{aligned}
& \int_{-L / 2}^{L / 2} \int_{-\pi}^{\pi}\left\{\varepsilon f(x) \cos (t-\phi / \omega)-\delta v_{B}-\sin \left(z_{1}+u_{B}\right)\right. \\
& \left.\quad+\sin \left(u_{B}\right)+\cos \left(u_{B}\right) z_{1}\right\} v_{B} d t d x=0,
\end{aligned}
$$

where $z=\left(z_{1}, z_{2}\right) . \partial_{t}(u, v)_{B}$ is the function spanning the one dimensional kernel of $A_{B}$. At $z=0$, we get the equation

$$
\varepsilon \sin (\phi / \omega) \int_{-L / 2}^{L / 2} \int_{-\pi}^{\pi} f(x) \sin (t) v_{B} d x d t=\delta \int_{-L / 2}^{L / 2} \int_{-\pi}^{\pi} v_{B}^{2} d x d t
$$

by expanding $\cos (t-\phi / \omega)$ and using that $\Theta$ is even with respect to sign reversal of both $x$ and $t$, see Date [D], this makes the $\cos (t)$ term drop out. [If for $L$ large we approximate the spatially periodic breather by the breather on the line

$$
u(x, t)=4 \tan ^{-1}\left[\frac{\alpha}{\omega} \frac{\cos (\omega t)}{\cos (\alpha x)}\right], \quad \alpha=\sqrt{1-\omega^{2}},
$$

for details of this see Grauer and Birnir [GB], then the above conditions reduce to

$$
\left.\varepsilon \sin (\phi / \omega) \int_{-L / 2}^{L / 2} \frac{f(x)}{\cosh (\alpha x)} d x=8 \delta .\right]
$$

The relationship (5.8) fixes the phase of the breather and shows that (5.6) is a necessary condition for the existence of a breather. It is also sufficient. The derivative of the second equation in (5.7), with respect to $\varepsilon$, is

$$
\sin (\phi / \omega) \int_{-L / 2}^{L / 2} \int_{-\pi}^{\pi} f(x) \sin (t) v_{B} d x d t \neq 0
$$

by the oddness of sine and $v_{B}$ in $t$, and the evenness in $x$ of $f$ and $\Theta$, at $z=0$, $\delta=0$. That the integral can only vanish at isolated values of $L$ is clear from the approximation for $L$ large above and the analyticity in $L$. Therefore, by the implicit function theorem there exists a smooth function $\varepsilon(z, \delta)$ in a neighborhood of $(\varepsilon, \delta, z)=0$, such that

$$
\Pi(N(z, \delta)+\varepsilon(z, \delta) f)=0 .
$$

The breathers are created by a saddle-node bifurcation, or there are two phases giving the same value of $\sin (\phi / \omega)$, one phase giving the stable, the other the unstable breather. 
There are two such pairs of breathers because the initial breather can either be centered in the middle or at the ends of the spatial interval, to be spatially symmetric.

We rewrite the first equation in (5.7), in integral form by use of a Greens function. The Greens function for the undamped operator $A_{B}(\delta=0)$ was computed in McLaughlin and Scott [MS]. It is formed by the functions in Lemma 5.1,

$$
\begin{aligned}
\mathscr{G}(x, t, r, \tau)= & -i \frac{\pi}{4} \sum_{n} \frac{\lambda_{n}\left(\lambda_{n}-\zeta_{1}^{-}\right)\left(\lambda_{n}-\zeta_{2}^{-}\right)}{\left(\lambda_{n}-\zeta_{1}^{+}\right)\left(\lambda_{n}-\zeta_{2}^{+}\right)} \\
& \times\left[\psi^{+}(x, t) \partial_{t} \psi^{-}(r, \tau)-\partial_{t} \psi^{+}(x, t) \phi^{-}(r, \tau)\right]\left(\begin{array}{cc}
0 & -1 \\
1 & 0
\end{array}\right),
\end{aligned}
$$

where $\zeta_{1}^{ \pm}=(\omega \mp i \alpha) / 4, \zeta_{2}^{ \pm}=-(\omega \mp i \alpha) / 4$. A Greens function for the damped operator is given by a similar formula using the functions in Lemma 5.2. However, we want to solve the first equations in (5.7) on kernel $A_{B}^{\perp}$, so we first exclude $g_{0}$ and then make the rest of the eigenfunction orthogonal to it. This is with respect to the inner product in $H^{1} \times L^{2}[0, L]$. Then the resulting Greens function gives the solution of (5.7),

$$
\begin{aligned}
y(t)= & \int_{L / 2}^{L / 2} G(x, t, r, 0) y(0) d r \\
& +\int_{0}^{t} \int_{L / 2}^{L / 2} G(x, t, r, \tau)(I-\Pi) N(s+y, \delta, \varepsilon) d r d \tau,
\end{aligned}
$$

where $N(z, \delta, \varepsilon)=N(z, \delta)+\varepsilon f$. The Poincaré map of (5.7) is

$$
y(t+2 \pi)=D P y(t)+\mathscr{N}^{\prime}(t, \delta, \varepsilon)
$$

where the Poincare map of the damped vector field linearized about the breather is

$$
D P y(t)=\int_{L / 2}^{L / 2} G(x, t+2 \pi, r, t) y(r, t) d r
$$

and

$$
\mathscr{N}(t, \delta, \varepsilon)=\int_{t}^{t+2 \pi} \int_{L / 2}^{L / 2} G(x, t+2 \pi, r, \tau)(I-\Pi) N(s+y, \delta, \varepsilon) d r d \tau
$$

lies in kern $A_{B}^{\perp} \subset H^{1} \times L^{2}[0, L]$. Now $y(t)$ is periodic in $t$, if and only if

$$
(I-D P) y(t)=\mathscr{N}(t, \delta, \varepsilon) .
$$

However, by Lemma 5.2, $\operatorname{kern}(I-D P)=\operatorname{kern} A_{B}$ is one dimensional and $(I-D P)^{-1}$ exist on $\operatorname{kern}(I-D P)^{\perp}$, because $\|D P\| \leq e^{-\delta T / 2}$ on $\operatorname{kern}(I-D P)^{\perp}$. Then we fix $\delta$ and apply the implicit function theorem to the equation

$$
y-(I-D P)^{-1} \mathcal{N}(y+s, \varepsilon)=0 .
$$




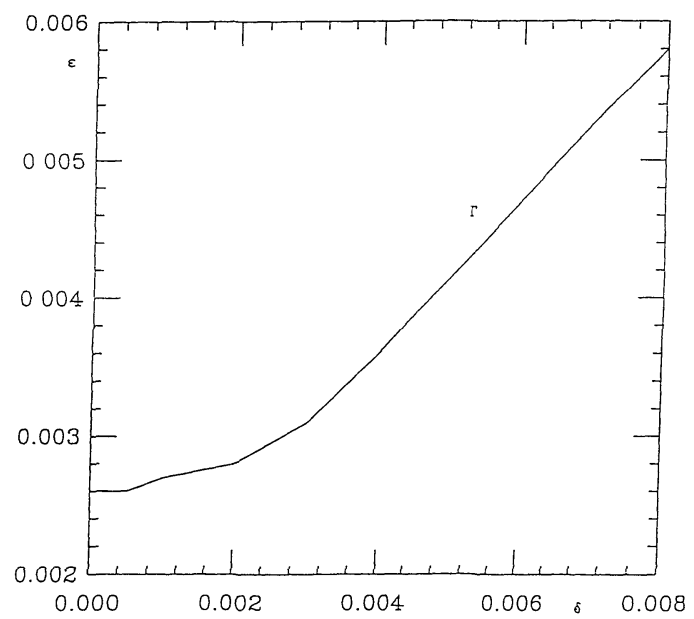

Fig. 5.1. The bifurcation curves of the breathers in the $\delta-\varepsilon$ plane

First, $N(0,0)=0$, so that $\mathscr{N}(0,0)=0$. Secondly,

$$
D_{y} N(s+y)=\left(\begin{array}{cc}
0 & 0 \\
-\cos \left(s_{1}+y_{1}+u_{B}\right)+\cos \left(u_{B}\right) & 0
\end{array}\right),
$$

so the Frechét derivative of $\mathscr{N}$ is bounded $\left\|D_{y} \mathscr{N}\right\| \leq 2\|G\|$, and $D_{y} \mathscr{N}(0,0)=0$. This implies that the Frechét derivative of (5.9) is $I \neq 0$, at $s=0=y, \varepsilon=0$. Consequently, by the implicit function theorem there exists a smooth function $y(s, \varepsilon)$ such that

$$
y(s, \varepsilon, t)=(I-D P)^{-1} \mathscr{N}(s+y(s, \varepsilon), \varepsilon),
$$

in a neighborhood of $(s, \varepsilon)=0$. The uniqueness of the solution of (5.7), for either the stable or the unstable breather, now follows because $\varepsilon$ is a function of $z=y(s)+s$, by the above, for $\delta$ fixed.

The actual curve $\Gamma$ in the $\delta-\varepsilon$ plane, above which the breathers exist is shown on Fig. 5.1. It is computed for $L=80$ and $f(x)=1$, in Condition 5.6.

We collect the pieces of the global picture, whose existence we have proven, in Table 5.1

Table 5.1

\begin{tabular}{lll}
\hline & Stable & Unstable \\
\hline Localized spatially & 2 breathers & 2 unstable breathers \\
Not spatially localized & The flat & $\begin{array}{l}\text { The straight-up } \\
3 n \text { flappers }\end{array}$ \\
\end{tabular}

The Global Attractor for Spatially Even Functions. We have seen that the $B$-attractor of the damped and driven sine-Gordon equation contains three components

$$
\mathscr{B}=\bigcup_{k=1}^{3} \mathscr{B}_{k}
$$


$\mathscr{P}_{1}$ is the stable flat, periodic orbit, $\mathscr{B}_{2}$ is the stable breather located at the center of the spatial interval, whereas $\mathscr{B}_{3}$ is the stable breather located at the ends of the interval. The "hyperbolic structure" on the other hand contains $3+3 n$, where $n=[L / 2 \pi]$ is the integer value, components

$$
\mathscr{C}=\bigcup_{k=1}^{3+3 n} \mathscr{C}_{k}+W_{k}^{u}
$$

$\mathscr{C}_{1}$ is the unstable straight-up periodic orbit, $\mathscr{C}_{2}$ and $\mathscr{C}_{3}$ are the unstable breathers located at the center and the ends of the spatial interval respectively, $\mathscr{C}_{k}, k=$ $4, \ldots, 3 n+3$, are the unstable flapper triplets created by the pitchfork bifurcation in Theorem 4.2. $W_{k}^{u}$ is the unstable manifold of $\mathscr{C}_{k}, k=1, \ldots, 3 n+3$.

The fractal dimension of . $t$ can be estimated, see Temam [T] and Babin and Vishik [BV], and to prove that

$$
\mathscr{A}=\mathscr{B} \cup \mathscr{C}
$$

we need to show that the lower count of dimension obtained from $\mathscr{B}$ and $\mathscr{C}$ agrees with a sharp upper estimate of the dimension of $\mathscr{C}$. Unfortunately, this is not a not a simple counting argument because the flapper triplets are not linearly independent and they span most of the six-dimensional space where the breathers reside, see Grauer and Birnir [GB]. However, for a range of $L$ the upper estimates agree with the lower ones, see Birnir [B4]. Below we will describe the numerical results which indicate that $\mathscr{C}=\mathscr{B} \cup \mathscr{C}$ for a large range of spatial intervals $(L)$. This is the only part of the paper that relies on numerics and an analytic proof will be given in Birnir [B4].

Numerical Results. Consider the parameter ranges, $45 \leq L \leq 200,0<\varepsilon, \delta<10^{-1}$, and let the driving function be $f=\cos (\omega t)$. Then the $B$-attractor consists of only the stable flat periodic orbit, below the curve $\Gamma$ in the $\delta-\varepsilon$ plane, on Fig. 5.1. Above this curve the $B$-attractor consists of the flat and the two stable breathers. The flat remains stable and time-periodic, but whereas the spatial profiles of the breathers remain the same, their temporal evolutions can become very complex due to bifurcations, see below, on a center manifold. The two breathers do not interact and evolve independently due to the spatial exponential decay. If more breathers are introduced through the initial data, they are destroyed and the solution goes to the flat. The basis of attraction of breathers is small whereas the basis of the flat is large. When $L>300$ the breathers cannot feel each other through their exponential tails and more than one pair can exist, showing identical bifurcations.

Now consider the parameter range $L \leq 45, \varepsilon, \delta>10^{-1}$. Then the two breathers interact but are not destroyed. This $B$-attractor was found and explored by Bishop et al [BM 1, 2, 3, 4]. A four mode truncation seems to give a qualitatively faithful picture of the dynamics of the PDE, see Bishop et al [3, 4]. Overman et al. [O], [MO] and Kovacic and Wiggins [Ko, KW]. In principle, this ought to be provable by the center-manifold reductions of Grauer and Birnir [GB] but the full bifurcation analysis of the PDE remains open.

Bifurcations of the Breathers. The stable breathers become marginal, and bifurcate as the ratio of the driving to the damping amplitudes $\varepsilon / \delta$ increases. We will now describe the bifurcations of spatially localized breathers; for $0<\varepsilon, \delta<10^{-1}$, they are summarized in Table 5.2 but Grauer and Birnir [GB] contains the details. We find three types of bifurcations, including two bifurcation sequences which are caused by a symmetry. In each case we start with a breather in the center, or the wings, whose 


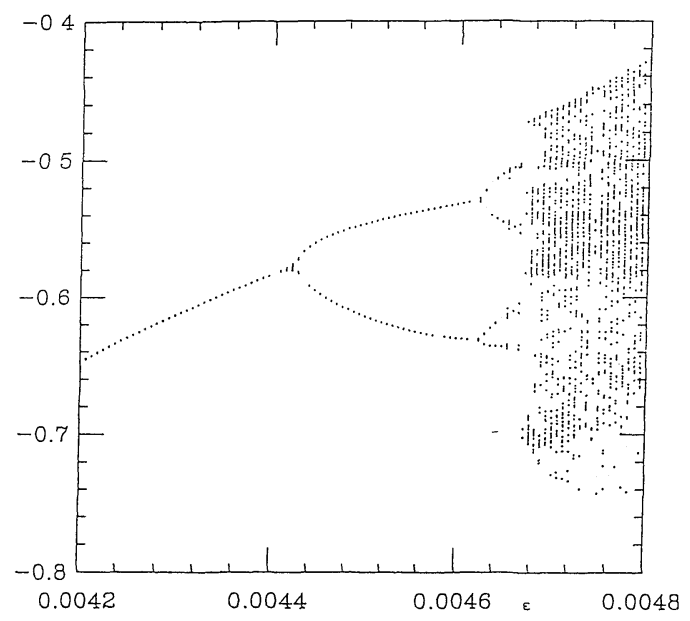

Fig. 5.2. The period doubling sequence of the breather-torus, $\delta=0.004, \omega=0.98, L=80$

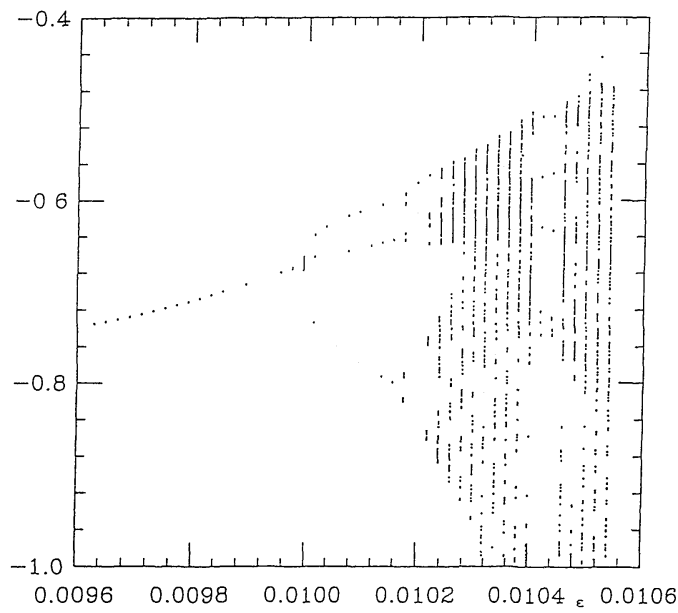

Fig. 5.3. The bifurcation sequence of the breather-torus showing quasi-periodicity and phaselocking, near period $3, \delta=0.004, \omega=0.96, L=80$

time behavior is periodic. That is to say, for $\varepsilon / \delta$ small but satisfying Condition 5.6, the breather is a periodic orbit in phase space. Then a Hopf bifurcations to a torus takes place, but thereafter there are three possibilities depending on $\omega$. We fix $L=80$, then if $\omega \geq 0.97$ a period doubling cascade of tori to a strange attractor takes place. It is exhibited in Fig. 5.2. On the other hand, if $0.93 \leq \omega \leq 0.97$, we get a 2nd Hopf bifurcation of the 2-torus to a 3-torus. As $\varepsilon / \delta$ increases there is a short (in $\varepsilon / \delta$ ) cascade of quasi-periodic orbits on 3-tori that culminates in a period tripling cascade to a strange attractor. The whole bifurcation sequence close to period 3 is shown in Fig. 5.3. In Fig. 5.4 the subregion where the cascade of quasi-periodic orbits takes place is enlarged. If $\omega \leq 0.93$, complicated transients are observed, but the solution eventually always goes to the flat. More details of all this are spelled out in Grauer and Birnir [GB]. 


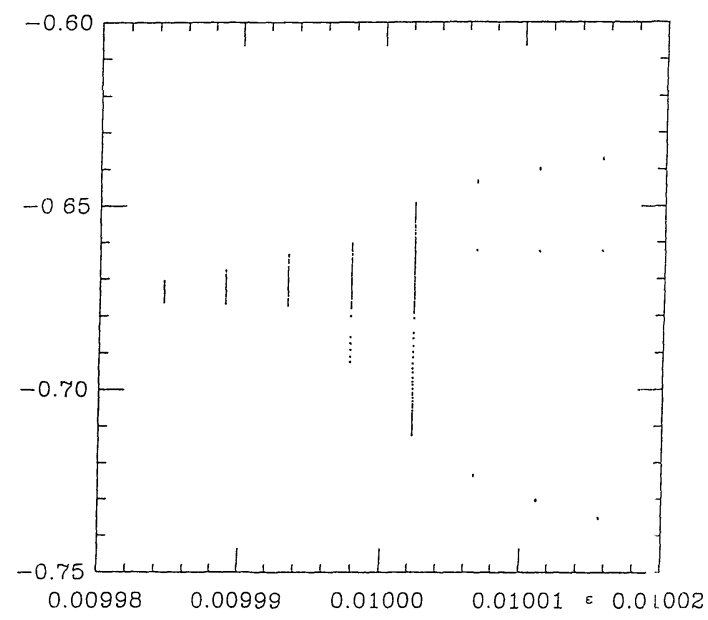

Fig. 5.4. A magnification of the cascade of quasi-periodic orbits in Fig. 5.3

Remark 5.1. Table 5.2 constitutes a very satisfying manifestation of the Feigenbaum and Ruelle-Takens theories for spatially localized solutions of PDE's. However, longtime chaotic evolution is localized in phase-space because it only occurs on the two separate breather-attractors. Moreover, the latter are spatially coherent so the situation is far from being "turbulent." Finally, the basin of attraction of the breathers is small compared to the flat, so most orbits are destined for the regular part (flat) of the $B$-attractor.

Table 5.2

\begin{tabular}{clll}
\hline$\omega$ & $1-.97$ & $.97-.93$ & $\leq .93$ \\
& Periodic orbit & Periodic orbit & Periodic orbit \\
& Hopf & Hopf & Hopf \\
$\varepsilon / \delta$ & 2-Torus & 2-Torus & The flat \\
$\downarrow$ & Period. doubling & 2nd Hopf & \\
& Strange attractor & 3-Torus & \\
& & Quasi-per. break-up & \\
& & Period tripling & \\
& & Strange attractor & \\
\hline
\end{tabular}

Conclusion. The damped and driven sine-Gordon equation has a finite-dimensional $A$-attractor $\mathscr{b}$. A complete qualitative analysis of the components of $\mathscr{b}$ is possible by use of modern analytic techniques. In fact these methods apply equally well to nonlinear wave equations and perhaps most dissipative PDE's on finite domains. The $A$-attractors decompose into a low-dimensional $B$-attractor $\mathscr{B}$ which attracts most of phase-space and a "hyperbolic structure" $\mathscr{C}$ which is associated with transient dynamics but can have a large dimension. This may resolve the apparent contradiction that estimates of $\operatorname{dim}$. A are typically large but numerically the long-time asymptotics seem to lie on a low-dimensional set, for most weakly-turbulent systems. 
The components of the $B$-attractor which are numerically verified to be all its components in the parameter region under consideration, are strikingly simple. They are a spatially homogeneous periodic orbit and the simplest spatially localized solutions possible: two breathers. Moreover, the bifurcations of the breathers are generic within the simple symmetries present.

When the spatial simplicity and temporal complexity of the $B$-attractor is compared with spatial complexity and temporal simplicity of the solutions to the completely integrable unperturbed sine-Gordon equation, one must conclude that there is little if any overlap. Compare Remark 4.3. This is not surprising considering the faith of the phase portrait of the nonlinear pendulum with added dissipation and remembering that there are infinitely many more directions to decay in, in function space.

It is matter of debate whether the attractor, above the $\varepsilon$ threshold $\Gamma$ in Fig. 5.1, has something to do with the integrable structure of the unperturbed sine-Gordon equation or not. Breathers exist above this line and the nonexistence of breathers result of Birnir, McKean and Weinstein [BMW], see also Segur and Kruskal [SK], can be interpreted to say that the PDE has to be integrable for breathers to exist, in the conservative case. We believe that the existence of these spatially localized solutions has more to do with the symmetry of the nonlinearity; that it is odd and permits a spatially localized hump. In fact, nonlinear wave equations which do not have breather solutions by Birnir, McKean and Weinstein [BMW] obtain breathers when the right amount of damping and driving is added, see Birnir and Nelson [BN], to the PDE.

\section{Appendix. The Center Manifold of the Flappers}

Proof of Proposition 4.4. We write the damped and driven sine-Gordon equation in the form

$$
\dot{w}=A w+N(w)+\varepsilon f,
$$

where $w=(u, v), N(w)=(0,-\sin (u)), f=(0, f(x, t))$, and

$$
A=\left(\begin{array}{cc}
0 & 1 \\
\partial_{x}^{2} & -\delta
\end{array}\right) \text {. }
$$

The equation linearized about a stationary flapper can be written

$$
\underline{\dot{w}}=A \underline{w}+N\left(w_{n}\right)+\varepsilon D_{w} N\left(w_{m}\right) \underline{w}_{3}+\varepsilon f,
$$

where $w_{m}=\left(u_{m}, v_{m}\right)$ is the $\left(m^{\text {th }}\right)$ flapper in Proposition 4.1 and $\underline{w}_{3}=\left(\underline{u}_{3}, \underline{v}_{3}\right)$ is the linear periodic orbit in Proposition 4.2. We let $z=w-\underline{w}$ and subtract the second equation from the first to get

$$
\dot{z}=A\left(u_{m}\right) z+N\left(z, \underline{w}_{3}\right),
$$

where

$$
A\left(u_{m}\right)=\left(\begin{array}{cc}
0 & 1 \\
\partial_{x}^{2}-\cos \left(u_{m}(x)\right) & -\delta
\end{array}\right)
$$

and

$$
N\left(z, \underline{w}_{3}\right)=\left(0,-\left[\sin \left(u_{m}\right)\left(z_{1}+\varepsilon \underline{u}_{3}\right)^{2} / 2+\cos \left(u_{m}\right)\left(z_{1}+\varepsilon \underline{u}_{3}\right)^{3} / 6\right]+\mathscr{O}(z, \varepsilon)^{4}\right) .
$$


By Lemma 4.1, $\sigma\left(A\left(u_{m}\right)\right.$ ) contains one eigenvalue $\lambda_{1}$ on the positive real axis, one eigenvalue $\lambda_{2}$ at 0 , and the remaining eigenvalues satisfy the bound

$$
\operatorname{Re} \lambda_{j} \leq-\delta / 2, \quad j \neq 0,1,2, \quad j \in \mathbb{Z} .
$$

Then by the center manifold theorem for flows, see for example Bates and Jones [BJ], there exist an unstable manifold $W^{u}$, a center manifold $W^{c}$ and a stable manifold $W^{s}$,

$$
\operatorname{dim} W^{u}=1=\operatorname{dim} W^{c}, \quad \operatorname{codim} W^{s}=2,
$$

of the stationary solution $(u, v)=\left(u_{m}, 0\right)$. We have to do the Floquet Theory of the flow on these manifolds to prove the existence of a periodic orbit and find its stability. First we compute the equations defining the flow on $W^{c}$. The operator $A\left(u_{m}\right)$ has the eigenvectors

$$
w_{1}=\left(\begin{array}{l}
1 \\
0
\end{array}\right) i d s \text { and } w_{2}=\left(\begin{array}{c}
1 \\
-\delta
\end{array}\right) i d s,
$$

corresponding to the eigenvalues 0 and $-\delta$ respectively and their dual vectors are

$$
w_{1}^{*}=\left(\begin{array}{c}
1 \\
1 / \delta
\end{array}\right) i d s \quad \text { and } \quad w_{2}^{*}=\left(\begin{array}{c}
0 \\
-1 / \delta
\end{array}\right) i d s
$$

where $d s$ is the Jacobi elliptic functions. This makes the projections onto $w_{1}$ and $w_{2}$ be

$$
\begin{aligned}
& \Pi_{1}(u, v)=\int_{0}^{L}(u+v / \delta) i d s d x\left(\begin{array}{l}
1 \\
0
\end{array}\right) i d s, \\
& \Pi_{2}(u, v)=-\int_{0}^{L}(v / \delta) i d s d x\left(\begin{array}{c}
1 \\
-\delta
\end{array}\right) i d s .
\end{aligned}
$$

Now let

$$
w=\left(a w_{1}+b w_{2}\right) i d s+y .
$$

Then $\Pi_{1}$ of (A.1) gives the ODE

$$
\dot{a}=-2 \varepsilon(a+b) \zeta-\varepsilon^{2} \xi+\mathscr{O}(a, b, \varepsilon)^{3}
$$

where

$$
\begin{aligned}
& \zeta=(1 / \delta) \int_{0}^{L}(i d s)^{\prime}(i d s)^{2} f(x) d x \cos (\omega t-\phi), \\
& \xi=(1 / \delta) \int_{0}^{\delta}(i d s)^{\prime}(i d s) f^{2}(x) d x \cos ^{2}(\omega t-\phi) .
\end{aligned}
$$

The coefficient of $(a+b)^{2}$ vanishes,

$$
\int_{0}^{L} i d s^{\prime}(i d s)^{3} d x=0
$$


by the periodicity of $d s$. Similarly, $\Pi_{2}$ of (A.1) gives the ODE

$$
\dot{b}=-\delta b-2 \varepsilon(a+b) \zeta-\varepsilon^{2} \xi+\odot(a, b, \varepsilon)^{3},
$$

and the equation for $y$ is

$$
\begin{aligned}
\dot{y}= & A\left(u_{m}\right) y+\left(I-\Pi_{1}-\Pi_{2}\right) \\
& \times\left(2 i d s^{\prime}((a+b) i d s+\varepsilon f(x) \cos (\omega t-\phi))^{2}+\mathscr{\sigma}(a, b, \varepsilon)^{3}\right),
\end{aligned}
$$

where we have used that $\sin \left(u_{m}\right)=2\left(\sqrt{1-n s^{2}}\right) n s=-2 i d s^{\prime}$. Now suppose that $f$ is an even function of $x$. Then $\zeta=0=\xi$ and we have to go to cubic order to compute the equation for $a$. The quadratic (in $a$ ) terms in $y$ contribute to the cubic term in

$$
\left(\begin{array}{c}
\dot{a} \\
0
\end{array}\right)=-\Pi_{1}\left(\begin{array}{c}
0 \\
\cos \left(u_{m}\right)((a+b) i d s+\varepsilon f \cos (\omega t-\phi))^{3} / 6 \\
+\sin \left(u_{m}\right)((a+b) i d s+\varepsilon f \cos (\omega t-\phi)) y_{1}
\end{array}\right) .
$$

We must know $y_{1}$ up to cubic terms to do the computation. By Lemma A.1,

$$
y=-2\left\{a^{2}\left(\begin{array}{c}
Q^{-1}(i d s)^{\prime}(i d s)^{2} \\
0
\end{array}\right)+\frac{\varepsilon^{2}}{2}\left(\begin{array}{c}
Q^{-1} f(x)(i d s)^{\prime} \\
0
\end{array}\right)\right\},
$$

where $Q=\partial_{x}^{2}-\cos \left(u_{m}\right)$. This is substituted into the above formula to get

$$
\dot{a}=\alpha a^{3}+\beta a^{2} \varepsilon \cos (\omega t-\phi)+\gamma a \varepsilon^{2} \cos ^{2}(\omega t-\phi)+\mu \varepsilon^{3} \cos ^{3}(\omega t-\phi),
$$

because $b(t)=b_{0} e^{-\delta t}+\mathscr{O}(a, b, \varepsilon)^{3}$ requires $b_{0}=0$ on $W^{c}$. The coefficients $\alpha, \beta, \gamma$, $\mu$ are collected in Table A. 1 ,

\section{Table A.1.}

$$
\begin{aligned}
& \alpha=(1 / \delta) \int_{0}^{L}\left[\frac{\left(2 n s^{2}-1\right)(i d s)^{4}}{6}+4(i d s)^{\prime}(i d s)^{2} Q^{-1}(i d s)^{\prime}(i d s)^{2}\right] d x \\
& \beta=(1 / \delta) \int_{0}^{L}\left[\frac{\left(2 n s^{2}-1\right)(i d s)^{3}}{2}+4(i d s)^{\prime}(i d s) Q^{-1}(i d s)^{\prime}(i d s)^{2}\right] f(x) d x \\
& \gamma=(1 / \delta) \int_{0}^{L}\left[\frac{\left(2 n s^{2}-1\right)(i d s)^{2}}{2}+2(i d s)^{\prime}(i d s)^{2} Q^{-1}(i d s)^{\prime}\right] f(x) d x \\
& \mu=(1 / \delta) \int_{0}^{L}\left[\frac{\left(2 n s^{2}-1\right)(i d s)}{6}+2(i d s)^{\prime}(i d s) Q^{-1}(i d s)^{\prime}\right] f(x) d x
\end{aligned}
$$

where we have used the identities

$$
\sin \left(u_{m}\right)=-2 i d s, \quad \cos \left(u_{m}\right)=2 n s^{2}-1 .
$$

We are jumping the gun here to get the values of the coefficients. The precise argument for computing $a$ and $y$ is given below. Finally the equation for $y$ follows if we notice that $\Pi_{k} N(z, w)=0, k=1,2$, up to cubic terms. 
Proof of Proposition 4.5. We start with the equation

$$
\begin{aligned}
\dot{a}= & -\frac{1}{\delta} \int_{0}^{L}\left[\cos \left(u_{m}\right)((a) i d s+\varepsilon f \cos (\omega t-\phi))^{3} / 6\right. \\
& \left.+\sin \left(u_{m}\right)((a) i d s+\varepsilon f \cos (\omega t-\phi)) y_{1}\right] i d s d x
\end{aligned}
$$

from the proof of Proposition 4.4, recalling that $b \equiv 0$ on $W^{c}$. We need to know $y_{1}$ up to cubic terms and by the center-manifold theorem $y_{1}$ can be expressed as

$$
y_{1}=a^{2} g_{1}+2 a \varepsilon g_{2}+\varepsilon^{3} g_{3}
$$

where $g_{\imath}(i=1,2,3)$ are functions of $x$ only. We substitute $y_{1}$ into the equation for $a$ and get the equation

$$
\begin{aligned}
\dot{a}-\frac{\gamma \varepsilon^{2}}{2} \cos (2(\omega t-\phi)) a= & \alpha a^{3}+\beta a^{2} \varepsilon \cos (\omega t-\phi) \\
& +\frac{\gamma a \varepsilon^{2}}{2}+\mu \varepsilon^{3} \cos ^{3}(\omega t-\phi),
\end{aligned}
$$

where the coefficients $\alpha, \beta, \gamma, \mu$ involve integrals over the unknown functions $g_{i}$ $(i=1,2,3)$.

The Floquet Theory of this equation is simple because it can be solved explicitly. The solution is

$$
\begin{aligned}
a= & a_{0} e^{c \varepsilon^{2} \sin (2(\omega t-\phi))} \\
+ & \int_{0}^{t}\left\{\left[\alpha a^{3}+\beta a^{2} \varepsilon \cos (\omega \tau-\phi)+\frac{\gamma a \varepsilon^{2}}{2}+\mu \varepsilon^{2} \cos ^{3}(\omega \tau-\phi)\right]\right. \\
& \left.\times e^{c \varepsilon^{2}[\sin (2(\omega t-\phi))-\sin (2(\omega \tau-\phi))]}\right\} d \tau
\end{aligned}
$$

where $c=-\gamma / 4 \omega$. This function is periodic in $t$ if

$$
\begin{aligned}
& \int_{0}^{T}\left[\alpha a^{3}+\beta a^{2} \varepsilon \cos (\omega t-\phi)+\frac{\gamma a \varepsilon^{2}}{2}+\mu \varepsilon^{2} \cos ^{3}(\omega t-\phi)\right] \\
& \quad \times e^{-c \varepsilon^{2}[\sin (2(T-\phi))-\sin (2(\omega t-\phi))]} d t=0 .
\end{aligned}
$$

A substitution of the leading order

$$
a=a_{0} e^{\left(c e^{2} \sin (2(\omega t-\phi))\right.}=a_{0}\left(1+c \varepsilon^{2} \sin (2(\omega t-\phi))+\mathscr{\odot}\left(\varepsilon^{4}\right)\right)
$$

into this condition, gives the equation

$$
\alpha a_{0}^{3}+\gamma \frac{a_{0} \varepsilon^{2}}{2}=0
$$

that is,

$$
a_{0}=0 \quad \text { or } \quad a_{0}= \pm \sqrt{\frac{-\gamma}{2 \alpha}} \varepsilon
$$


The form of $a$

$$
a=a_{0}\left(1+c \varepsilon^{2} \sin (2(\omega t-\phi))+\mathscr{Q}\left(\varepsilon^{4}\right)\right.
$$

now suffices to compute $y$ up to cubic order. In other words, we compute $g_{\imath}$ $(i=1,2,3)$ in Lemma A.1, and this determines $\alpha, \beta, \gamma, \mu$, see Table A.1.

Now

$$
\alpha=(1 / \delta) \int_{0}^{L}\left[\frac{\left(2 n s^{2}-1\right)(i d s)^{4}}{6}+4(i d s)^{\prime}(i d s)^{2} Q^{-1}(i d s)^{\prime}(i d s)^{2}\right] d x<0
$$

because

$$
\max n s^{2}=K^{2}=\frac{8 \pi n}{L}\left(1-\frac{2 \pi n}{L}\right) \leq 1, \quad \text { if } \quad L \approx 2 \pi n,
$$

in addition, $n s$ does have a localized peak and $d s=0$, where $n s=K$. Thus,

$$
\int_{0}^{L} \frac{\left(2 n s^{2}-1\right)(i d s)^{4}}{6} d x<0 .
$$

Moreover, $i d s^{\prime} i d s^{2}$ is perpendicular to the eigenfunctions of $Q$ with positive and zero eigenvalues,

$$
\int_{0}^{L} i d s^{\prime} i d s^{3} d x=0=\int_{0}^{L} i d s i d s^{2} i c s d x
$$

and all the other eigenvalues of $Q$ are negative. The upshot is that,

$$
\int_{0}^{L}(i d s)^{\prime}(i d s)^{2} Q^{-1}(i d s)^{\prime}(i d s)^{2} d x<0 .
$$

The constant

$$
\gamma=(1 / \delta) \int_{0}^{L}\left[\frac{\left(2 n s^{2}-1\right)(i d s)^{2}}{2}+2(i d s)^{\prime}(i d s)^{2} Q^{-1}(i d s)^{\prime}\right] f(x) d x
$$

can either be positive or negative depending on $f$. When $\gamma<0$, we get a supercritical pitchfork bifurcation with two stable and one unstable branches. When $\gamma>0$, we get a subcritical pitchfork bifurcation with one stable branch.

The upshot is that we get three branches bifurcating from $a=0$, if $\gamma<0$ and one branch if $\gamma>0$. In particular, $f=1$ gives $\gamma<0$.

The existence and smoothness in $\varepsilon$, of these branches, follows from the implicit function theorem in $\mathbb{R}^{2}$, because

and

$$
\begin{gathered}
\left.\frac{\partial^{3}}{\partial a^{3}} \Pi_{1} N(x+y, w)\right|_{a=0}=\alpha<0, \\
\left.\frac{\partial^{3}}{\partial a \partial^{2} \varepsilon} \Pi_{1} N(x+y, w)\right|_{\substack{a=0 \\
\varepsilon=0}}=\gamma \neq 0,
\end{gathered}
$$

by Table A.1. 
The $a_{0}=0$ branch is unstable and the $a_{0}= \pm \sqrt{\frac{-\gamma}{2 \alpha}} \varepsilon$ branches are stable, for $\gamma<0$. For $\gamma>0$, there is only one stable branch $a_{0}=0$.

Lemma A.1. The infinite-dimensional part $y$, of the solution in Proposition 4.4 is time-periodic if and only if

$$
y=-2\left\{a^{2}\left(\begin{array}{c}
Q^{-1}(i d s)^{\prime}(i d s)^{2} \\
0
\end{array}\right)+\frac{\varepsilon^{2}}{2}\left(\begin{array}{c}
Q^{-1} i d s^{\prime} f(x) \\
0
\end{array}\right)\right\}+\mathscr{Q}(a, \varepsilon)^{3} .
$$

Proof. We let

$$
y=a^{2}\left(\begin{array}{l}
g_{1} \\
h_{1}
\end{array}\right)+2 a \varepsilon\left(\begin{array}{l}
g_{2} \\
h_{2}
\end{array}\right)+\varepsilon^{3}\left(\begin{array}{l}
g_{3} \\
h_{3}
\end{array}\right)
$$

and substitute into the $y$ equation in Proposition 4.4. Then $y$ is the $T$-periodic if

$$
\begin{aligned}
0= & \int_{0}^{T} \dot{y} d t=\int_{0}^{T}\left\{\left[a^{2}\left(\begin{array}{c}
h_{1} \\
Q g_{1}-\delta h_{1}
\end{array}\right)+2 a \varepsilon\left(\begin{array}{c}
h_{2} \\
Q g_{2}-\delta h_{2}
\end{array}\right)\right.\right. \\
& \left.+\varepsilon^{2}\left(\begin{array}{c}
h_{3} \\
Q g_{3}-\delta h_{3}
\end{array}\right)\right]+2\left[a^{2}\left(\begin{array}{c}
0 \\
i d s^{\prime}(i d s)^{2}
\end{array}\right)\right. \\
& \left.\left.+2 a \varepsilon\left(\begin{array}{c}
0 \\
i d s^{\prime} i d s f
\end{array}\right) \cos (\omega t-\phi)+\varepsilon^{2}\left(\begin{array}{c}
0 \\
i d s^{\prime} f^{2}
\end{array}\right) \cos ^{2}(\omega t-\phi)\right]\right\} d t
\end{aligned}
$$

where $Q=\partial_{x}^{2}-\cos \left(u_{m}\right)$. By Proposition 4.5 the above formula for $y$ implies that $a$ has the form

$$
a=a_{0}\left(1+c \varepsilon^{2} \sin (2(\omega t-\phi))+\mathscr{O}\left(\varepsilon^{4}\right)\right),
$$

and we obtain the formula for $y$ by substituting this expression in for $a$.

\section{References}

[A] Arnold, V.I.: Ordinary differential equations. Boston: MIT Press, 1973

[ABB] Armbruster, D., Birnir, B., Buys, M.: The damped and driven sine-Gordon equation and basic turbulence. UCSB preprint (1993)

[B1] Birnir, B.: Complex Hill's equation and the complex periodic Korteweg-de Vries equation. Comm. Pure Applied Math. 39, 1-49 (1986)

[B2] Birnir, B.: Singularities of the complex KdV flows. Comm. Pure and Applied Math. 39, 283-305 (1986)

[B3] Birnir, B.: An example of finite-time blow-up of the complex Korteweg-de Vries equation and existence beyond blow-up. SIAM J. Appl. Math. 47, 710-725 (1987)

[B4] Birnir, B.: Dimension estimates of attractors of nonlinear dissipative wave equations with applications to the damped and driven sine-Gordon attraction. In preparation

[BF1] Bishop, A.R., Forest, M.G., McLaughlin, D.W., Overman, E.A. II: A quasi-periodic route to chaos in a near integrable PDE. Physica D 23, 293-328 (1986)

[BF2] Bishop, A.R., Forest, M.G., McLaughlin, D.W., Overman, E.A. II: Quasi-periodic route to chaos in a near integrable PDE, homoclinic crossings. Phys. Lett. A 127, 335-340 (1988)

[BF3] Bishop, A.R., Forest, M.G., McLaughlin, D.W., Overman, E.A. II: A modal representation of chaotic attractors for the driven damped pendulum chain. Phys. Lett. A 144, 17-25 (1990)

[BF4] Bishop, A.R., Flesch, R., Forest, M.G., McLaughlin, D.W., Overman, E.A. II: Correlations between chaos in a perturbed sine-Gordon equation and a truncated model system. SIAM J. Math. Anal., 1511-1536 (1990) 
[BJ] Bates, P., Jones, C.K.R.T.: Invariant manifolds for semilinear partial differential equations. Dynamics Reported 2, 1-39 (1989)

[BMW] Birnir, B., McKean, H., Weinstein, A.: Nonexistence of breathers. CPAM, to appear

[BN] Birnir, B., Nelson, K.: Global attractors of periodically forced nonlinear dissipative wave equations. In preparation

[BS] Birnir, B., Smereka, P.: Existence theory and invariant manifolds of driven bubble clouds. Comm. Pure Appl. Math. 43, 363-413 (1990)

[BV] Babin, A.V. Vishik, M.I.: Attractors of evolution equations. Studies in Appl. Math. and its Applic. Vol. 25. Amsterdam: North-Holland, 1992

[C] Carr, J.: Applications of center manifold theory. Berlin, Heidelberg, New York: Springer 1981

[CH] Chow, S.-N., Hale, J.K.: Methods of Bifurcation Theory. Berlin, Heidelberg, New York: Springer, 1982

[CL] Coddington, E.A., Levinson, N.: Theory of ordinary differential equations. New York: McGraw-Hill, 1955

[D] Date, E.: Multisoliton solutions and quasi-periodic solutions of nonlinear equations of sine-Gordon type. Osaka J. Math. 19, 125-128 (1982)

[GT] Ghidaglia, J.M., Temam, R.: Attractors for damped nonlinear hyperbolic equations. J. Math pures and appl. 66, 273-319 (1987)

[GB] Grauer, R., Birnir, B.: The center manifold and bifurcations of the sine-Gordon breather. Physica D56, 165-184 (1992)

[H] Hartman, P.: Ordinary differential equations. New York: Willey, 1964

[Ha] Hale, J.: Asymptotic behavior of dissipative systems. Providence: AMS, 1988

[K] Kuratowski, K.: Topology, Vol. 1. New York: Academic Press 1966

[Ko] Kovacic, G.: Chaos in a model of the forced and damped sine-Gordon equation. Ph.D. thesis, California Institute of Technology, 1989

[KW] Kovacic, G., Wiggins, S.: Orbits homoclinic to resonances, with an application to chaos in a model of the forced and damped sine-Gordon equation. Physica D, to appear

[L] Lions, J.L.: Quelques methods de résolution des problèmes aux limites nonlinéaires. Paris: Dunod, 1969

[LS] Lomdahl, P.S., Samuelsen, M.R.: Persistent breather excitations in an AC-driven sineGordon system with loss. Phys. Rev. Lett. 34, 664 (1986)

[M] Mañé, R.: Dynam. Syst. and turbulence. Warwick, ed. Rand, D.A. and Young, L.-S., Berlin, Heidelberg, New York: Springer, 1980

[McK] McKean, H.: The sine-Gordon and sinh-Gordon equation on the circle. Comm. Pure Appl. Math. 34, 197-243 (1981)

[Mi] Milnor, J.: On the concept of attractor. Commun. Math. Phys. 99, 177-195 (1985)

[MB] Mazor, A., Bishop, A.R., McLaughlin, D.W.: Phase-puling and space-time complexity in an ac driven damped one-dimensional sine-Gordon system. Phys. Lett. A 119, 273-279 (1986)

[MO] McLaughlin, D.W., Overman, E.A., II: Whiskered tori for integrable PDEs and chaotic behavior in near integrable PDEs, Surveys in Appl. Math. 1 (1992)

[MS] McLaughlin, D.W., Scott, A.C.: Perturbation analysis of fluxon dynamics. Phys. Rev. A 28, 1652-1680 (1978)

[MW] Magnus, W., Wrinkler, W.: Hill's equation. New York: Willey-Interscience, 1966

[NM] Nayfeh, A.H., Mook, D.T.: Nonlinear oscillation. New York: Willey 1979

[O] Overman, E., Xiong, C., Berliner, M.: Low mode truncation methods in the sine-Gordon equation. Preprint, Ohio State University, 1991

[P] Pedersen, N.F.: Advances in superconductivity, ed. Deaver and Ruvolds. New York: Plenum, 1982

[PV] du Val, P.: Elliptic functions and elliptic curves. Cambridge: Cambridge Univ. Press, 1973

[R] Reed, M.: Abstract non-linear wave eqs. Lecture Notes in Math. 507. Berlin, Heidelberg, New York: Springer, 1976

[RF] Reinish, G., Fernandez, J.C., Goupil, M.J., Legrand, O.: Relativistic dynamics of sineGordon solitons trapped in confining potentials. Phys. Rev. B. 34, 6207 (1986)

[S] Siegel, C.L.: Topics in Complex Function Theory. Vol. 1-3, New York: Willey-Interscience, 1971 
[SK] Segur, H., Kruskal, M.D.: Nonexistence of small amplitude breather solutions in $\phi^{4}$ theory. Phys. Rev. Lett. 58, 747-750 (1987)

[SYC] Sauer, T., York, J.A., Casdagli, M.: Embedology. Stat. Phys. 65: 3-4, 579-617 (1991)

[T] Temam, R.: Infinite-dimensional dynamical systems in mechanics and physics. Berlin, Heidelberg, New York: Springer 1988

[Ta] Taki, M., Spatschek, K.H., Fernandez, J.C., Grauer, R., Reinisch, G.: Breather dynamics in the nonlinear Schrödinger regime of perturbed sine-Gordon systems. Physica D $\mathbf{4 0}$, 65-84 (1989)

[TF] Takhatajan, L.A., Faddeev, L.D.: Essentially nonlinear one-dimensional model of classical field theory. Theor. Math. Phys. 21, 1046-1156

Communicated by T. Spencer 